\title{
Frequency-Shift Zero-Forcing Time-Varying Equalization for Doubly Selective SIMO Channels
}

\begin{abstract}
Francesco Verde
Dipartimento di Ingegneria Elettronica e delle Telecomunicazioni, Università degli Studi di Napoli Federico II, via Claudio 21, 80125 Napoli, Italy
\end{abstract}

Received 1 June 2005; Revised 26 February 2006; Accepted 30 April 2006

\begin{abstract}
This paper deals with the problem of designing linear time-varying (LTV) finite-impulse response zero-forcing (ZF) equalizers for time- and frequency-selective (so-called doubly selective) single-input multiple-output (SIMO) channels. Specifically, relying on a basis expansion model (BEM) of the rapidly time-varying channel impulse response, we derive the canonical frequency-domain representation of the minimal norm LTV-ZF equalizer, which allows one to implement it as a parallel bank of linear time-invariant filters having, as input signals, different frequency-shift (FRESH) versions of the received data. Moreover, on the basis of this FRESH representation, we propose a simple and effective low-complexity version of the minimal norm LTV-ZF equalizer and we discuss the relationships between the devised FRESH equalizers and a LTV-ZF equalizer recently proposed in the literature. The performance analysis, carried out by means of computer simulations, shows that the proposed FRESH-LTV-ZF equalizers significantly outperform their competitive alternative.
\end{abstract}

Copyright ( 2006 Hindawi Publishing Corporation. All rights reserved.

\section{INTRODUCTION}

In many wireless applications, such as high-speed Internet access, networking, digital audio, and video broadcasting, the increasing need to provide either high data-rate services for low-mobility users or low data-rate services for highmobility users has made Doppler spreading and intersymbol interference the main performance limiting factors.

The design of low-complexity reliable detection strategies for wireless communication systems operating over timeand frequency-selective, so-called doubly selective, channels requires an accurate description of the time-varying behaviour of the transmission media. Several approaches for modeling finite-impulse response (FIR) linear time-varying (LTV) channels have been developed in the last decade (see [1] for a comprehensive review). Among all the others, deterministic basis expansion models (BEMs) [2-6] seem to be favoured for representing rapidly time-varying channels, since they offer well-structured parsimonious modeling of channel time variations. Specifically, BEMs allow one to express the channel impulse response as a superposition of time-varying complex exponentials with time-invariant coefficients. As pointed out in [1,7], BEMs with complex exponentials approximate well the Jakes statistical model [8], which is widely adopted for simulating wireless communication channels.
Recently, relying on a BEM to represent doubly selective channels, serial and block FIR-LTV equalizers have been developed in [9], which are synthesized by resorting to both zero-forcing (ZF) and minimum mean-square error (MMSE) criteria. In particular, it was argued in [9] that since a doubly selective channel cannot be diagonalized by a channel-independent transformation, the implementation of block LTV equalizers, which collect and process in blocks all the available data in the received frame, leads to an unsustainable computational complexity. On the other hand, serial LTV equalizers, which process few data at a time, exhibit a good tradeoff between complexity and performance. Given these considerations, in this paper, we focus attention exclusively on serial LTV equalizers and, in particular, we consider only ZF solutions. This last choice is motivated by the fact that, for many modulation formats, the bit error rate performances of $\mathrm{ZF}$ equalizers can be evaluated in closed form in the presence of additive white Gaussian noise (AWGN), without resorting to any approximation; moreover, the performances of MMSE equalizers strongly depend on the existence of ZF solutions. Before [9], the synthesis of LTV-ZF serial equalizers for doubly selective channels has been considered in $[1,10]$. In these papers, however, the authors have derived only an implicit time-domain representation of the equalizer weight vector, without fully exploiting the particular time variation of the BEM either for 
synthesis of LTV-ZF equalizers or for discussing the mathematical conditions assuring their existence. On the contrary, the authors in [9] have provided an explicit frequencydomain representation of a LTV-ZF equalizer, by turning a challenging LTV equalization problem in a simpler linear time-invariant (LTI) filtering design, which involves only the time-invariant coefficients of the BEM of the doubly selective channel.

In this paper, borrowing concepts from the well-known theory of polyperiodic linear filtering [11], we provide a unified framework to design LTV-ZF equalizers for doubly selective channels. In particular, we derive the canonical frequency-domain representation of the minimal norm LTV-ZF equalizer, which leads to an implementation configuration having an embedded predominant time-invariant component. In this representation, the time-varying component of the minimal norm LTV-ZF equalizer consists solely of computing frequency-shift (FRESH) versions of the received data. The main advantage of the FRESH representation of the minimal norm LTV-ZF equalizer is threefold: (i) it allows us to establish many similarities to LTI conventional equalization techniques; (ii) it allows us to individuate a simple and effective low-complexity suboptimal implementation of the minimal norm LTV-ZF equalizer; (iii) it allows us to provide an alternative interpretation of the LTV-ZF equalizer proposed in [9], by showing in particular that the frequencydomain representation derived in [9] is not canonical and, moreover, does not lead to the minimal norm LTV-ZF equalizer.

The paper is organized as follows. In Section 2, we introduce the BEM of the wireless doubly selective channel and discuss the assumptions that hold throughout the paper. In Section 3, the time-domain representation of the minimal norm LTV-ZF equalizer is introduced, and the mathematical conditions assuring its existence are discussed. In Section 4, we derive the frequency-domain representation of the minimal norm LTV-ZF equalizer and synthesize its lowcomplexity suboptimal implementation. In Section 5, we discuss in depth the relationships between the proposed approaches and the previously proposed technique [9]. Section 6 provides numerical results, obtained by means of Monte Carlo simulations, aimed at assessing the performances of the proposed FRESH equalization algorithms and comparing them with those of [9]. Concluding remarks are drawn in Section 7.

\subsection{Basic notations}

Upper- and lower-case bold letters denote matrices and vectors; the superscripts $*, T, H,-1,-$, and $\dagger$ denote the conjugate, the transpose, the Hermitian (conjugate transpose), the inverse, the generalized (1)-inverse [12], and the MoorePenrose generalized inverse [12] of a matrix; $\mathbb{C}, \mathbb{R}$, and $\mathbb{Z}$ are the fields of complex, real, and integer numbers; $\mathbb{C}^{n}\left[\mathbb{R}^{n}\right]$ denotes the vector-space of all $n$-column vectors with complex [real] coordinates; similarly, $\mathbb{C}^{n \times m}\left[\mathbb{R}^{n \times m}\right]$ denotes the vector-space of all the $n \times m$ matrices with complex [real] elements; $\mathbf{0}_{n}, \mathbf{O}_{n \times m}$, and $\mathbf{I}_{n}$ denote the $n$-column zero vector, the $n \times m$ zero matrix, and the $n \times n$ identity matrix; $\|\mathbf{a}\|$ denotes the Frobenius norm of any vector $\mathbf{a} ; \operatorname{rank}(\mathbf{A}), \mathcal{N}(\mathbf{A})$, and $\{\mathbf{A}\}_{i, j}$ indicate the rank, the null space, and the $(i, j)$ th entry of any matrix $\mathbf{A} ; \mathbf{A}=\operatorname{diag}\left[\mathbf{A}_{11}, \mathbf{A}_{22}, \ldots, \mathbf{A}_{n n}\right]$ is the (block) diagonal matrix wherein $\left\{\mathbf{A}_{i i}\right\}_{i=1}^{n}$ are the block diagonal entries; $\operatorname{vec}(\mathbf{A})$ associates with any matrix $\mathbf{A}$ the vector obtained by stacking its columns, $\mathrm{E}[\cdot]$ denotes statistical averaging; $\langle a(k)\rangle_{K} \triangleq(1 / K) \sum_{k=k_{0}}^{k_{0}+K-1} a(k)$ denotes temporal averaging of the arbitrary sequence $a(k)$ over the time interval $\left\{k_{0}, k_{0}+1, \ldots, k_{0}+K-1\right\}$, with $k_{0} \in \mathbb{Z}$; and, finally, $(\cdot)_{P},\lceil\cdot\rceil$, $\otimes$, and $j \triangleq \sqrt{-1}$ denote modulo- $P$ operation, integer ceiling, Kronecker product, and imaginary unit.

\section{THE MATHEMATICAL MODEL}

Let us consider a single-input multiple-output (SIMO) digital communication system, equipped with one transmitter antenna and $N$ receiver antennas, employing linear modulation with baud-rate $1 / T_{s}$ and transmitting over a double selective channel. The complex envelope of the received signal at the $n$th antenna, after filtering, ideal carrier-frequency recovering, and baud-rate sampling, can be expressed as

$$
r_{n}(k)=\sum_{\ell=0}^{L_{h}} h_{n}(k, \ell) s(k-\ell)+v_{n}(k),
$$

where $s(k)$, with $k \in \mathbb{Z}$, is the sequence of the transmitted symbols, $h_{n}(k, \ell)$ denotes the composite impulse response (including transmitting filter, physical channel, and receiving filter) of the LTV (discrete-time) channel corresponding to the $n$th receiver antenna, which is assumed to be a causal FIR filter of order $L_{h}>0$, and $v_{n}(k)$ is additive noise at the output of the receiving filter employed at the $n$th antenna. The following assumptions will be considered throughout the paper:

(A1) the information symbols $s(k)$ are modeled as ${ }^{1}$ a QPSK sequence of independent and identically distributed (i.i.d.) random variables assuming equiprobable values in $8=\{ \pm 1 / \sqrt{2}, \pm j / \sqrt{2}\}$

(A2) the noise samples $\left\{v_{n}(k)\right\}_{n=1}^{N}$ are modeled as mutually independent i.i.d. complex circular zero-mean Gaussian random sequences, with variance $\sigma_{v}^{2} \triangleq$ $\mathrm{E}\left[\left|v_{n}(k)\right|^{2}\right]$, independent of $s(k)$.

Let us consider a $K$-length observation window $\mathcal{K} \triangleq$ $\left\{k_{0}, k_{0}+1, \ldots, k_{0}+K-1\right\}$, with $k_{0} \in \mathbb{Z}$ denoting an arbitrary time instant, we rely in this paper on the following BEM [6] of the $n$th time-varying channel:

$$
\begin{aligned}
h_{n}(k, \ell)=\sum_{q=-Q_{h} / 2}^{Q_{h} / 2} h_{q, n}(\ell) e^{j(2 \pi / P) q k}, & \text { for } k \in \mathcal{K}, \ell \in\left\{0,1, \ldots, L_{h}\right\},
\end{aligned}
$$

\footnotetext{
${ }^{1}$ This assumption is made only for the sake of simplicity and all the results derived in the sequel can be straightforwardly extended to other linear modulation formats.
} 
wherein the time-varying behaviour of the transmission media is represented by means of $Q_{h}+1$ harmonically related complex exponentials with a frequency spacing of $2 \pi / P$. In this representation, $h_{q, n}(\ell)$ are deterministic coefficients ${ }^{2}$ and $Q_{h} \triangleq 2\left\lceil f_{\max } P T_{s}\right\rceil$, where $f_{\max }$ denotes the Doppler spread of the channel defined as in [13]. Note that, in comparison with previously proposed BEMs (see, e.g., [4]), wherein $h_{n}(k, \ell)$ is represented over the $K$-length observation window $\mathcal{K}$ as a linear combination of complex exponentials with a frequency spacing of $2 \pi / K$, that is, $P=K$, the BEM considered in (2) employs complex exponentials with a smaller frequency spacing of $2 \pi / P \leq 2 \pi / K$, that is, $P \geq K$. As it is shown in [6], reducing the frequency spacing between the complex exponentials allows one to obtain more accurate representations of $h_{n}(k, \ell)$ over the whole observation interval $\mathcal{K}$. The channel model (2) is particularly useful for the receiver synthesis. First, since it turns out that $f_{\max } T_{s} \ll 1$ for many practical systems and, hence, $Q_{h} \ll P$, model (2) is a parsimonious representation of the time-varying transmission channel. Second, since both $L_{h}$ and $f_{\max }$ can be measured experimentally, model (2) shows that, for $k \in \mathcal{K}$, the $n$th time-varying channel $h_{n}(k, \ell)$ is unknown up to only time-invariant scalars $h_{q, n}(\ell)$, which can be estimated blindly $[1,10,14]$ or by employing training sequences $[15,16]$. In this paper, we assume that, for each antenna, the coefficients $\left\{h_{q, n}(\ell)\right\}_{q=-Q_{h} / 2}^{Q_{h} / 2}$ are perfectly known at the receiver, for all $\ell \in\left\{0,1, \ldots, L_{h}\right\}$.

Finally, accounting for (2) and collecting the samples $\left\{r_{n}(k)\right\}_{n=1}^{N}$ received by the $N$ antennas into the vector $\mathbf{r}(k) \triangleq$ $\left[r_{1}(k), r_{2}(k), \ldots, r_{N}(k)\right]^{T} \in \mathbb{C}^{N}$, we obtain the compact SIMO vector model

$\mathbf{r}(k)=\sum_{\ell=0}^{L_{h}} \sum_{q=-Q_{h} / 2}^{Q_{h} / 2} \mathbf{h}_{q}(\ell) s(k-\ell) e^{j(2 \pi / P) q k}+\mathbf{v}(k), \quad$ for $k \in \mathcal{K}$,

where $\mathbf{h}_{q}(\ell) \triangleq\left[h_{q, 1}(\ell), h_{q, 2}(\ell), \ldots, h_{q, N}(\ell)\right]^{T} \in \mathbb{C}^{N}$ and $\mathbf{v}(k) \triangleq$ $\left[v_{1}(k), v_{2}(k), \ldots, v_{N}(k)\right]^{T} \in \mathbb{C}^{N}$.

\section{LINEAR TIME-VARYING ZERO-FORCING EQUALIZATION}

In order to compensate for the channel-induced impairments, we consider a causal LTV equalizer of order $L_{e}>0$, whose output $y(k)$ can be written in vector form as

$$
y(k)=\mathbf{f}^{H}(k) \mathbf{z}(k)
$$

where the vector $\mathbf{f}(k) \in \mathbb{C}^{N\left(L_{e}+1\right)}$ collects all the equalizer parameters whereas, by virtue of (3), the input vector $\mathbf{z}(k) \triangleq$ $\left[\mathbf{r}^{T}(k), \mathbf{r}^{T}(k-1), \ldots, \mathbf{r}^{T}\left(k-L_{e}\right)\right]^{T} \in \mathbb{C}^{N\left(L_{e}+1\right)}$ can be explicitly

\footnotetext{
2 It is worth noting that although the coefficients $h_{q, n}(\ell)$ are allowed to change with $k_{0}$, for the sake of notation simplicity, we do not explicitly indicate the dependence of $h_{q, n}(\ell)$ on $k_{0}$.
}

expressed as

$$
\mathbf{z}(k)=[\sum_{q=-Q_{h} / 2}^{Q_{h} / 2} \underbrace{\mathbf{J}_{q} \mathbf{H}_{q}}_{\widetilde{\mathbf{H}}_{q}} e^{j(2 \pi / P) q k}] \mathbf{s}(k)+\mathbf{w}(k), \quad \text { for } k \in \mathcal{K},
$$

with

$$
\begin{gathered}
\mathbf{J}_{q} \triangleq \operatorname{diag}\left[\mathbf{I}_{N}, e^{-j(2 \pi / P) q} \mathbf{I}_{N}, \ldots, e^{-j(2 \pi / P) q L_{e}} \mathbf{I}_{N}\right] \\
\in \mathbb{C}^{N\left(L_{e}+1\right) \times N\left(L_{e}+1\right)}, \\
\mathbf{s}(k) \triangleq\left[s(k), s(k-1), \ldots, s\left(k-L_{e}-L_{h}\right)\right]^{T} \in \mathbb{C}^{L_{e}+L_{h}+1}, \\
\mathbf{w}(k) \triangleq\left[\mathbf{v}^{T}(k), \mathbf{v}^{T}(k-1), \ldots, \mathbf{v}^{T}\left(k-L_{e}\right)\right]^{T} \in \mathbb{C}^{N\left(L_{e}+1\right)},
\end{gathered}
$$

and, moreover, we have defined the block Toeplitz matrices

$$
\begin{aligned}
& \mathbf{H}_{q} \triangleq\left[\begin{array}{ccccccc}
\mathbf{h}_{q}(0) & \cdots & \mathbf{h}_{q}\left(L_{h}\right) & \mathbf{0}_{N} & \mathbf{0}_{N} & \cdots & \mathbf{0}_{N} \\
\mathbf{0}_{N} & \mathbf{h}_{q}(0) & \cdots & \mathbf{h}_{q}\left(L_{h}\right) & \mathbf{0}_{N} & \cdots & \mathbf{0}_{N} \\
\vdots & \ddots & \ldots & \ddots & \ddots & \cdots & \vdots \\
\mathbf{0}_{N} & \cdots & \mathbf{0}_{N} & \mathbf{0}_{N} & \mathbf{h}_{q}(0) & \cdots & \mathbf{h}_{q}\left(L_{h}\right)
\end{array}\right] \\
& \in \mathbb{C}^{N\left(L_{e}+1\right) \times\left(L_{e}+L_{h}+1\right)} .
\end{aligned}
$$

Our aim is to reliably estimate the transmitted symbol $s(k-$ $d)$, with $d \in\left\{0,1, \ldots, L_{e}+L_{h}\right\}$ denoting a suitable equalization delay. To this goal, we focus attention exclusively on $\mathrm{ZF}$ designs of the equalizer weight vector $\mathbf{f}(k)$. In Sections 3.1 and 3.2, the time-domain representation of the minimumnorm LTV-ZF equalizer is discussed.

\subsection{Time-domain representation of the minimum-norm LTV-ZF equalizer}

As it can be seen from (4) and (5), in the absence of noise, imposing the ZF condition $y(k)=s(k-d)$ leads to the following system of linear equations:

$$
\mathbf{f}^{H}(k) \underbrace{\left[\sum_{q=-Q_{h} / 2}^{Q_{h} / 2} \widetilde{\mathbf{H}}_{q} e^{j(2 \pi / P) q k}\right]}_{\widetilde{\mathbf{H}}(k)}=\mathbf{f}^{H}(k) \tilde{\mathbf{H}}(k)=\mathbf{e}_{d}^{T}, \quad \text { for } k \in \mathcal{K},
$$

with $\mathbf{e}_{d} \triangleq[\overbrace{0, \ldots, 0}^{d}, 1,0, \ldots, 0]^{T} \in \mathbb{R}^{L_{e}+L_{h}+1}$. This system is consistent [12] if and only if $\widetilde{\mathbf{H}}^{H}(k)\left[\tilde{\mathbf{H}}^{H}(k)\right]^{-} \mathbf{e}_{d}=\mathbf{e}_{d}$, for all $k \in \mathcal{K}$. If the time-varying matrix

$$
\widetilde{\mathbf{H}}(k) \in \mathbb{C}^{N\left(L_{e}+1\right) \times\left(L_{e}+L_{h}+1\right)}
$$


is full-column rank, that is, $\operatorname{rank}[\tilde{\mathbf{H}}(k)]=L_{e}+L_{h}+1$, for all $k \in \mathcal{K}$, it results that $\widetilde{\mathbf{H}}^{H}(k)\left[\tilde{\mathbf{H}}^{H}(k)\right]^{-}=\mathbf{I}_{L_{e}+L_{h}+1}$, for all $k \in \mathcal{K}$, and, then, the system (10) turns out to be consistent independently of the equalization delay $d$. In this case, the minimal norm solution of (10), that is, the solution of the constrained optimization problem

$$
\mathbf{f}_{\text {opt }}(k)=\arg \min _{\mathbf{f}(k)}\|\mathbf{f}(k)\|^{2}, \quad \text { subject to } \widetilde{\mathbf{H}}^{H}(k) \mathbf{f}(k)=\mathbf{e}_{d},
$$

is given by (see, e.g., [12])

$$
\begin{aligned}
\mathbf{f}_{\mathrm{opt}}(k) & =\left[\tilde{\mathbf{H}}^{H}(k)\right]^{\dagger} \mathbf{e}_{d} \\
& =\widetilde{\mathbf{H}}(k)\left[\tilde{\mathbf{H}}^{H}(k) \tilde{\mathbf{H}}(k)\right]^{-1} \mathbf{e}_{d}, \quad \text { for } k \in \mathcal{K} .
\end{aligned}
$$

Before providing sufficient conditions assuring the existence of LTV-ZF equalizers, it is useful to derive, in the presence of noise, the expression of the average bit error rate (ABER) for an arbitrary equalizer $\mathbf{f}(k)$ satisfying the LTV-ZF condition (10). We define the ABER of the detected symbol block as follows:

$$
\mathrm{ABER} \triangleq\left\langle P_{e}(k)\right\rangle_{K},
$$

where $P_{e}(k)$ denotes the bit error probability associated with the detection of the $k$ th symbol $s(k-d)$, for $k \in \mathcal{K}$. By invoking assumptions (A1) and (A2), it can be shown [17] that

$$
P_{e}(k)=Q\left(\frac{1}{\sigma_{v}\|\mathbf{f}(k)\|}\right), \quad \text { for } k \in \mathcal{K},
$$

where $Q(x) \triangleq(1 / \sqrt{2 \pi}) \int_{x}^{+\infty} e^{-u^{2} / 2} d u$ denotes the $Q$ function. Relations (12), (14) and (15) show that, in the presence of AWGN, the minimal norm equalizer $\mathbf{f}_{\text {opt }}(k)$ is optimal, in the sense that it achieves the minimum ABER among all the LTV equalizers satisfying the ZF condition (10). In the sequel, we refer to (13) as the time-domain representation of the optimal LTV-ZF equalizer $\mathbf{f}_{\text {opt }}(k)$.

Since the condition $\operatorname{rank}[\tilde{\mathbf{H}}(k)]=L_{e}+L_{h}+1$, for all $k \in$ $\mathcal{K}$, assures the consistency of the system (10) and, thus, the existence of LTV-ZF equalizers, it seems natural now to investigate the rank properties of $\tilde{\mathbf{H}}(k)$. On this subject, we provide the following theorem.

Theorem 1 (existence of LTV-ZF equalizers). For a given in$\operatorname{dex} q \in\left\{-Q_{h} / 2,-Q_{h} / 2+1, \ldots, Q_{h} / 2\right\}$, let $z_{q, 1}, z_{q, 2}, \ldots, z_{q, M_{q}}$ denote the $0 \leq M_{q} \leq L_{h}$ common zeros of the $N$ channel transfer functions $H_{q, n}(z)=\sum_{\ell=0}^{L_{h}} h_{q, n}(\ell) z^{-\ell}$ associated with the sequences $\left\{h_{q, n}(\ell)\right\}_{\ell=0}^{L_{h}}$, for $n \in\{1,2, \ldots, N\}$. Then, the timevarying matrix $\widetilde{\mathbf{H}}(k)$ is full-column rank, for all $k \in \mathbb{Z}$, if the following conditions are satisfied:

(C1) $N\left(L_{e}+1\right) \geq L_{e}+L_{h}+1$;

(C2) $z_{q_{1}, n_{1}} \neq z_{q_{2}, n_{2}}$, for all $q_{1} \neq q_{2} \in\left\{-Q_{h} / 2,-Q_{h} / 2+\right.$ $\left.1, \ldots, Q_{h} / 2\right\}$ and for all $n_{1} \neq n_{2} \in\{1,2, \ldots, N\}$.
Proof. See Appendix A.

It is worth noting that, similarly to the results assuring identifiability of linear time-invariant channels [18], condition $(\mathrm{C} 1)$ requires a minimum number $N_{\min }=2$ of receiver antennas. Moreover, for a fixed number of antennas $N \geq$ $N_{\min }$, the minimum value $L_{e, \min }$ of the equalizer order $L_{e}$ is given by $L_{e, \min }=L_{h} /(N-1)-1$, which does not depend on the number $Q_{h}+1$ of complex exponentials employed in (2) to represent the $N$ time-varying channels; in particular, for $N=N_{\min }$, one has $L_{e, \min }=L_{h}-1$. Finally, observe that condition (C2) imposes a very mild constraint on the time-invariant channels $\left\{h_{q, n}(\ell)\right\}_{\ell=0}^{L_{h}}$, for $q \in\left\{-Q_{h} / 2\right.$, $\left.-Q_{h} / 2+1, \ldots, Q_{h} / 2\right\}$ and $n \in\{1,2, \ldots, N\}$. Specifically, if the polynomials $H_{q, 1}(z), H_{q, 2}(z), \ldots, H_{q, N}(z)$ have no common zeros, that is, $M_{q}=0$, for all $q \in\left\{-Q_{h} / 2,-Q_{h} / 2+1, \ldots\right.$, $\left.Q_{h} / 2\right\}$, then the matrix $\tilde{\mathbf{H}}(k)$ turns out to be full-column rank, for all $k \in \mathbb{Z}$. More generally, the matrix $\widetilde{\mathbf{H}}(k)$ is fullcolumn rank, for all $k \in \mathbb{Z}$, even when, for a given index $q_{1} \in\left\{-Q_{h} / 2,-Q_{h} / 2+1, \ldots, Q_{h} / 2\right\}$, the channel transfer functions $H_{q_{1}, 1}(z), H_{q_{1}, 2}(z), \ldots, H_{q_{1}, N}(z)$ have $0<M_{q_{1}} \leq L_{h}$ common zeros, provided that, for all $n \in\{1,2, \ldots, N\}$, the complex number $z_{q_{1}, n}$ is not a common zero of $H_{q_{2}, 1}(z)$, $H_{q_{2}, 2}(z), \ldots, H_{q_{2}, N}(z)$, for all $q_{2} \neq q_{1} \in\left\{-Q_{h} / 2,-Q_{h} / 2+\right.$ $\left.1, \ldots, Q_{h} / 2\right\}$. Hereinafter, it is assumed that conditions $(\mathrm{C} 1)$ and (C2) are fulfilled.

\subsection{Implementation issues}

Let us return to the synthesis of the optimal LTV-ZF equalizer given by (13). To obtain an estimate of the transmitted block of symbols, one has to build the time-varying matrix $\widetilde{\mathbf{H}}(k)$ and, then, to compute the equalizer weight vector $\mathbf{f}_{\text {opt }}(k)$ for each value of $k \in \mathcal{K}$, by performing various mathematical operations on $\widetilde{\mathbf{H}}(k)$, such as, matrix multiplications and inversions. Therefore, notwithstanding its simple form, the time-domain implementation of $\mathbf{f}_{\text {opt }}(k)$ may lead to a high run-time complexity (in terms of floating point operations, subscripting, and memory traffic), especially for large values of the block size $K$.

In Section 4, by fully exploiting the particular time variation of the channel model (2), we derive the frequencydomain representation of $\mathbf{f}_{\text {opt }}(k)$, which represents the timefrequency dual of the time-domain representation (13) and allows us to show that the implementation of the optimal LTV-ZF equalizer can be obtained by resorting to LTI filtering of frequency-shifted versions of the received vector $\mathbf{z}(k)$.

\section{FREQUENCY-DOMAIN REPRESENTATION OF THE OPTIMAL LTV-ZF EQUALIZER}

Although the LTV-ZF condition given by (10) is valid only for $k \in \mathcal{K}$, without loss of generality, it is mathematically convenient for the synthesis of $\mathbf{f}(k)$ to regard (10) as a condition defined for all values of $k \in \mathbb{Z}$, but where only the $K$ values $\mathbf{f}\left(k_{0}\right), \mathbf{f}\left(k_{0}+1\right), \ldots, \mathbf{f}\left(k_{0}+K-1\right)$ of the synthesized $\mathbf{f}(k)$ will be used for producing the equalizer outputs $y(k)$, for $k \in \mathcal{K}$. 


\subsection{FRESH representation of the optimal LTV-ZF equalizer}

As a first step, accounting for (10), let us equivalently express the time-varying matrix $\widetilde{\mathbf{H}}(k)$ as follows:

$$
\widetilde{\mathbf{H}}(k)=\sum_{p=0}^{P-1} \mathscr{H}_{p} e^{j(2 \pi / P) p k}
$$

where we have defined the (matrix-valued) sequence

$$
\mathscr{H}_{p} \triangleq\left\{\begin{array}{lr}
\tilde{\mathbf{H}}_{p}, & \text { for } p \in\left\{0,1, \ldots, Q_{h} / 2\right\} \\
\mathbf{O}_{N\left(L_{e}+1\right) \times\left(L_{h}+L_{e}+1\right)}, & \text { for } p \in\left\{Q_{h} / 2+1, Q_{h} / 2\right. \\
& \left.+2, \ldots, P-Q_{h} / 2-1\right\} \\
\tilde{\mathbf{H}}_{p-P}, & \text { for } p \in\left\{P-Q_{h} / 2, P-Q_{h} / 2\right. \\
& +1, \ldots, P-1\} .
\end{array}\right.
$$

Note that, when $k \in \mathbb{Z}$, relation (16) can be regarded as the discrete Fourier series (DFS) expansion of the periodically time-varying matrix $\tilde{\mathbf{H}}(k)$, that is, $\tilde{\mathbf{H}}(k+P)=\widetilde{\mathbf{H}}(k)$, where the Fourier coefficients $\mathscr{H}_{p}$ can be interpreted to be a sequence of finite length $P$, given by (17) for $p \in\{0,1, \ldots, P-$ $1\}$, and zero otherwise. Consequently, the minimal norm vector $\mathbf{f}(k)$ satisfying the LTV-ZF condition (10), for all $k \in$ $\mathbb{Z}$, turns out to be also periodic with period $P$ and, thus, it can be expressed by means of its DFS representation:

$$
\mathbf{f}(k)=\sum_{p=0}^{P-1} \mathbf{f}_{p} e^{j(2 \pi / P) p k}
$$

where the $P$-length sequence $\left\{\mathbf{f}_{p}\right\}_{p=0}^{P-1}$ represent the Fourier coefficients of $\mathbf{f}(k)$. By substituting (16) and (18) in (10), after straightforward manipulations, one obtains the equivalent form

$$
\sum_{p=0}^{P-1}\left[\sum_{m=0}^{P-1} \mathscr{H}_{(m-p) P}^{H} \mathbf{f}_{m}\right] e^{j(2 \pi / P) p k}=\mathbf{e}_{d}
$$

which comes from the property that the Fourier coefficients of a product of two periodic sequences is the circular convolution of their respective Fourier coefficients. Since identity (19) must hold for all values of $k \in \mathbb{Z}$, and the complex exponentials in (19) are linearly independent functions, we can equate factors of corresponding exponential terms, obtaining thus the following $P$ systems of linear equations:

$$
\sum_{m=0}^{P-1} \mathcal{H}_{(m-p) P}^{H} \mathbf{f}_{m}= \begin{cases}\mathbf{e}_{d}, & \text { for } p=0, \\ \mathbf{0}_{L_{e}+L_{h}+1}, & \text { for } p \in\{1,2, \ldots, P-1\},\end{cases}
$$

which can be concisely written in matrix form as follows:

$$
\mathscr{H}_{\text {circ }}^{H} \psi=J_{d},
$$

where

$$
\mathscr{H}_{\mathrm{circ}} \triangleq\left[\begin{array}{ccccc}
\mathscr{H}_{0} & \mathscr{H}_{P-1} & \cdots & \mathscr{H}_{2} & \mathscr{H}_{1} \\
\mathscr{H}_{1} & \mathscr{H}_{0} & \cdots & \mathscr{H}_{3} & \mathscr{H}_{2} \\
\vdots & \vdots & \vdots & \vdots & \vdots \\
\mathscr{H}_{P-1} & \mathscr{H}_{P-2} & \cdots & \mathscr{H}_{1} & \mathscr{H}_{0}
\end{array}\right] \in \mathbb{C}^{N P\left(L_{e}+1\right) \times P\left(L_{e}+L_{h}+1\right)}
$$

is a block circulant [19] matrix, the vector $\psi \triangleq\left[\mathbf{f}_{0}^{T}, \mathbf{f}_{1}^{T}, \ldots\right.$, $\left.\mathbf{f}_{P-1}^{T}\right]^{T} \in \mathbb{C}^{N P\left(L_{e}+1\right)}$ collects all the Fourier coefficients of $\mathbf{f}(k)$, and, finally, $J_{d} \triangleq\left[\mathbf{e}_{d}^{T}, \mathbf{0}_{L_{e}+L_{h}+1}^{T}, \ldots, \mathbf{0}_{L_{e}+L_{h}+1}^{T}\right]^{T} \in \mathbb{R}^{P\left(L_{e}+L_{h}+1\right)}$. Note that (21) represents the equivalent frequency-domain representation of the time-domain LTV-ZF condition (10) and, moreover, it can be shown that, under conditions (C1) and (C2), the block circulant matrix $\mathscr{H}_{\text {circ }}$ is full-column rank. At this point, we note that, using the method of Lagrange multipliers, the potential solutions of the constrained optimization problem (12) are all vectors $\mathbf{f}(k)$ satisfying the linear system

$$
\mathbf{f}(k)+\tilde{\mathbf{H}}(k) \boldsymbol{\lambda}(k)=\mathbf{0}_{N\left(L_{e}+1\right)},
$$

where, when $k \in \mathbb{Z}$, the Lagrange multiplier $\boldsymbol{\lambda}(k) \in \mathbb{C}^{L_{e}+L_{h}+1}$ turns out to be periodically time-varying with period $P$, whose DFS expansion is given by

$$
\lambda(k)=\sum_{p=0}^{P-1} \lambda_{p} e^{j(2 \pi / P) p k}
$$

with the $P$-length sequence $\left\{\boldsymbol{\lambda}_{p}\right\}_{p=0}^{P-1}$ representing the Fourier coefficients of $\lambda(k)$. The periodically time-varying nature of $\lambda(k)$ can be readily proven from (23) by observing that, since $\mathbf{f}(k)$ and $\tilde{\mathbf{H}}(k)$ are periodically time-varying with period $P$, one has $\mathbf{f}(k+P)+\widetilde{\mathbf{H}}(k+P) \boldsymbol{\lambda}(k+P)=\mathbf{f}(k)+\tilde{\mathbf{H}}(k) \boldsymbol{\lambda}(k+P)=$ $\mathbf{0}_{N\left(L_{e}+1\right)}$, for all $k \in \mathbb{Z}$, which, accounting again for (23), implies that $\tilde{\mathbf{H}}(k) \boldsymbol{\lambda}(k+P)=\tilde{\mathbf{H}}(k) \boldsymbol{\lambda}(k)$ or, equivalently, $\tilde{\mathbf{H}}(k)[\boldsymbol{\lambda}(k)-\boldsymbol{\lambda}(k+P)]=\mathbf{0}_{N\left(L_{e}+1\right)}$. Since $\tilde{\mathbf{H}}(k)$ is full-column rank for any $k \in \mathbb{Z}$ (see Theorem 1 ), this matrix equation admits the unique solution $\lambda(k)=\lambda(k+P)$, for all $k \in \mathbb{Z}$. By substituting (16), (18), and (24) in (23), and reasoning as previously done, it can be verified that the equivalent frequency-domain representation of (23) can be expressed as

$$
\boldsymbol{\psi}+\mathscr{H}_{\mathrm{circ}} \boldsymbol{\chi}=\mathbf{0}_{N P\left(L_{e}+1\right)}
$$

where the vector $\boldsymbol{\chi} \triangleq\left[\boldsymbol{\lambda}_{0}^{T}, \boldsymbol{\lambda}_{1}^{T}, \ldots, \boldsymbol{\lambda}_{P-1}^{T}\right]^{T} \in \mathbb{C}^{P\left(L_{e}+L_{h}+1\right)}$ collects all the Fourier coefficients of $\lambda(k)$. By solving (25) with respect to vector $\psi$ and substituting the result into the constraint (21), one obtains $\chi_{\mathrm{opt}}=-\left(\mathscr{H}_{\text {circ }}^{H} \mathscr{H}_{\text {circ }}\right)^{-1} J_{d}$ which, in its turn, can be substituted in (25), obtaining thus

$$
\psi_{\mathrm{opt}} \triangleq\left[\begin{array}{c}
\mathbf{f}_{0, \mathrm{opt}} \\
\mathbf{f}_{1, \mathrm{opt}} \\
\vdots \\
\mathbf{f}_{P-1, \mathrm{opt}}
\end{array}\right]=\left(\mathscr{H}_{\mathrm{circ}}^{H}\right)^{\dagger} J_{d}=\mathscr{H}_{\mathrm{circ}}\left(\mathscr{H}_{\mathrm{circ}}^{H} \mathscr{H}_{\mathrm{circ}}\right)^{-1} J_{d}
$$




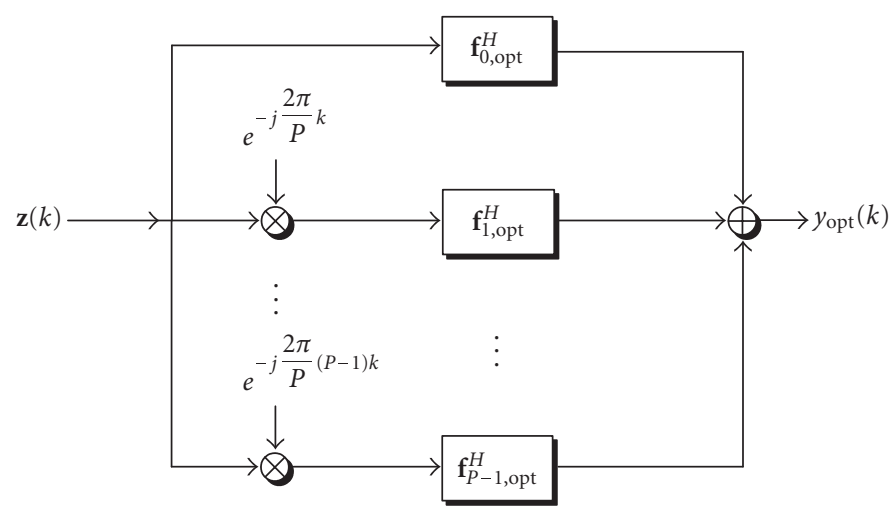

FIGURE 1: FRESH representation of the minimal norm LTV-ZF equalizer.

Remarkably, relation (26) shows that the equivalent frequency-domain representation of $\mathbf{f}_{\text {opt }}(k)$ turns out to be the minimal norm solution of the frequency-domain LTV-ZF condition (21), that is,

$$
\psi_{\mathrm{opt}}=\arg \min _{\psi}\|\psi\|^{2}, \quad \text { subject to } \mathscr{H}_{\mathrm{circ}}^{H} \psi=J d \text {. }
$$

Furthermore, accounting for (4), (18), and (26), the output $y_{\text {opt }}(k)$ of the optimal LTV-ZF equalizer $\mathbf{f}_{\text {opt }}(k)$ can be written as

$$
\begin{aligned}
y_{\mathrm{opt}}(k) & =\mathbf{f}_{\mathrm{opt}}^{H}(k) \mathbf{z}(k)=\left[\sum_{p=0}^{P-1} \mathbf{f}_{p, \mathrm{opt}} e^{j(2 \pi / P) p k}\right]^{H} \mathbf{z}(k) \\
& =\psi_{\mathrm{opt}}^{H} \widetilde{\mathbf{z}}(k), \quad \text { for } k \in \mathcal{K}
\end{aligned}
$$

where, by virtue of (5) and (22), the extended vector

$$
\tilde{\mathbf{z}}(k) \triangleq \zeta(k) \otimes \mathbf{z}(k) \in \mathbb{C}^{N P\left(L_{e}+1\right)},
$$

with $\zeta(k) \triangleq\left[1, e^{-j(2 \pi / P) k}, \ldots, e^{-j(2 \pi / P)(P-1) k}\right]^{T} \in \mathbb{C}^{P}$, can be explicitly written as

$$
\widetilde{\mathbf{z}}(k)=\mathscr{H}_{\text {circ }} \widetilde{\mathbf{s}}(k)+\widetilde{\mathbf{w}}(k)
$$

where $\tilde{\mathbf{s}}(k) \triangleq \boldsymbol{\zeta}(k) \otimes \mathbf{s}(k) \in \mathbb{C}^{P\left(L_{e}+L_{h}+1\right)}$ and $\tilde{\mathbf{w}}(k) \triangleq \boldsymbol{\zeta}(k) \otimes$ $\mathbf{w}(k) \in \mathbb{C}^{N P\left(L_{e}+1\right)}$. Relations (28) and (30) describe the FRE$\mathrm{SH}$ representation [11] of $\mathbf{f}_{\text {opt }}(k)$, wherein the minimal norm LTV-ZF equalizer is represented in the frequency-domain as a parallel bank of LTI equalizers, each one of them is driven by a different frequency-shifted version of $\mathbf{z}(k)$, and the output $y_{\text {opt }}(k)$ is formed by summing the outputs of the equalizers. A graphical representation for this parallel configuration is sketched in Figure 1.

\subsection{Explicit expression of the optimal Fourier coefficients}

At first sight, it may seem that the inversion of the large matrix $\mathscr{H}_{\text {circ }}^{H} \mathscr{H}_{\text {circ }} \in \mathbb{C}^{P\left(L_{e}+L_{h}+1\right) \times P\left(L_{e}+L_{h}+1\right)}$ must be performed in order to obtain the optimal Fourier coefficients given by (26). Interestingly, we show that, by suitably exploiting the block circulant nature of $\mathscr{H}_{\text {circ }}$, the optimal Fourier coefficients can be instead obtained by performing only inversions of $\left(L_{e}+L_{h}+1\right)$-dimensional square matrices. To see this, we preliminarily observe that, by using (22), the matrix $\mathscr{H}_{\text {circ }}^{H} \mathscr{H}_{\text {circ }}$ turns out to be also block circulant having the form

$$
\begin{aligned}
\mathscr{H}_{\text {circ }}^{H} & \mathscr{H}_{\text {circ }} \\
= & {\left[\begin{array}{ccccc}
\mathbf{A}_{0} & \mathbf{A}_{1} & \cdots & \mathbf{A}_{P-2} & \mathbf{A}_{P-1} \\
\mathbf{A}_{P-1} & \mathbf{A}_{0} & \cdots & \mathbf{A}_{P-3} & \mathbf{A}_{P-2} \\
\vdots & \vdots & \vdots & \vdots & \vdots \\
\mathbf{A}_{1} & \mathbf{A}_{2} & \cdots & \mathbf{A}_{P-1} & \mathbf{A}_{0}
\end{array}\right] \in \mathbb{C}^{P\left(L_{e}+L_{h}+1\right) \times P\left(L_{e}+L_{h}+1\right)}, }
\end{aligned}
$$

where $\mathbf{A}_{q} \triangleq \sum_{p=0}^{P-1} \mathscr{H}_{p}^{H} \mathscr{H}_{(p-q) P} \in \mathbb{C}^{\left(L_{e}+L_{h}+1\right) \times\left(L_{e}+L_{h}+1\right)}$. Accounting for (17) and observing that the matrices $\left\{\mathbf{A}_{q}\right\}_{q=0}^{P-1}$ are Hermitian symmetric, that is, $\mathbf{A}_{q}=\mathbf{A}_{(P-q)_{P}}^{H}$, one obtains

$$
\mathbf{A}_{q}=\left\{\begin{array}{lc}
\sum_{p=0}^{P-1} \mathcal{H}_{p}^{H} \mathcal{H}_{(p-q) P}, & \text { for } q \in\left\{0,1, \ldots, Q_{h}\right\}, \\
\mathbf{O}_{\left(L_{h}+L_{e}+1\right) \times\left(L_{h}+L_{e}+1\right)}, & \text { for } q \in\left\{Q_{h}+1, Q_{h}+2, \ldots, P\right. \\
& \left.-Q_{h}-1\right\}, \\
\mathbf{A}_{P-q}^{H}, & \text { for } q \in\left\{P-Q_{h}, P-Q_{h}\right. \\
& +1, \ldots, P-1\},
\end{array}\right.
$$

which shows that, practically, one has to evaluate only $Q_{h}+1$ of the $P$ matrices $\left\{\mathbf{A}_{q}\right\}_{q=0}^{P-1}$. At this point, we exploit the fact that the inverse of a block circulant matrix is again block circulant exhibiting a very nice structure [20]. Specifically, for the problem at hand, we have that the inverse $\left(\mathscr{H}_{\text {circ }}^{H} \mathscr{H}_{\text {circ }}\right)^{-1}$ is a block circulant matrix of the form

$$
\begin{aligned}
& \left(\mathscr{H}_{\text {circ }}^{H} \mathscr{H}_{\text {circ }}\right)^{-1} \\
& =\left[\begin{array}{ccccc}
\mathbf{B}_{0} & \mathbf{B}_{1} & \cdots & \mathbf{B}_{P-2} & \mathbf{B}_{P-1} \\
\mathbf{B}_{P-1} & \mathbf{B}_{0} & \cdots & \mathbf{B}_{P-3} & \mathbf{B}_{P-2} \\
\vdots & \vdots & \vdots & \vdots & \vdots \\
\mathbf{B}_{1} & \mathbf{B}_{2} & \cdots & \mathbf{B}_{P-1} & \mathbf{B}_{0}
\end{array}\right] \in \mathbb{C}^{P\left(L_{e}+L_{h}+1\right) \times P\left(L_{e}+L_{h}+1\right)},
\end{aligned}
$$


where $\mathbf{B}_{q} \triangleq\left(1 / P^{2}\right) \sum_{p=0}^{P-1} \mathcal{B}_{p}^{-1} e^{j(2 \pi / P) p q} \in \mathbb{C}^{\left(L_{e}+L_{h}+1\right) \times\left(L_{e}+L_{h}+1\right)}$, with $\mathcal{B}_{p} \triangleq(1 / P) \sum_{q=0}^{P-1} \mathbf{A}_{q} e^{-j(2 \pi / P) p q}$ representing the discrete Fourier transform (DFT) of the matrices $\left\{\mathbf{A}_{q}\right\}_{q=0}^{P-1}$. Accounting for (32), these DFT matrices can be explicitly written as

$$
\mathcal{B}_{p}=\frac{1}{P}\left(\mathbf{A}_{0}+\Delta_{p}+\Delta_{p}^{H}\right), \quad \text { for } p \in\{0,1, \ldots, P-1\},
$$

where

$$
\boldsymbol{\Delta}_{p} \triangleq \sum_{q=1}^{Q_{h}} \mathbf{A}_{q} e^{-j(2 \pi / P) p q} \in \mathbb{C}^{\left(L_{e}+L_{h}+1\right) \times\left(L_{e}+L_{h}+1\right)}
$$

On the basis of (34), the matrices $\left\{\mathbf{B}_{q}\right\}_{q=0}^{P-1}$ assume the final form:

$$
\begin{aligned}
& \mathbf{B}_{q} \triangleq \frac{1}{P} \sum_{p=0}^{P-1}\left(\mathbf{A}_{0}+\Delta_{p}+\Delta_{p}^{H}\right)^{-1} e^{j(2 \pi / P) p q}, \\
& \qquad \text { for } q \in\{0,1, \ldots, P-1\} .
\end{aligned}
$$

It is interesting to note that the computation of the matrices $\left\{\mathbf{B}_{q}\right\}_{q=0}^{P-1}$ requires the inversion of only the small matrices $\mathbf{A}_{0}+\Delta_{p}+\Delta_{p}^{H} \in \mathbb{C}^{\left(L_{e}+L_{h}+1\right) \times\left(L_{e}+L_{h}+1\right)}$, for $p \in\{0,1, \ldots, P-1\}$. Moreover, observe that, since $\mathbf{A}_{0}$ is a Hermitian matrix, the matrices $\left\{\mathbf{B}_{q}\right\}_{q=0}^{P-1}$ turn out to be Hermitian symmetric, that is, $\mathbf{B}_{q}=\mathbf{B}_{(P-q)_{P}}^{H}$ and, consequentially, in order to construct the inverse $\left(\mathscr{H}_{\text {circ }}^{H} \mathscr{H}_{\text {circ }}\right)^{-1}$, one has to evaluate in practice only $^{3} P / 2+1$ of the $P$ matrices $\left\{\mathbf{B}_{q}\right\}_{q=0}^{P-1}$. At this point, by substituting (22) and (33) in (26), and accounting moreover for (17), it can be shown that, after some algebraic manipulations, the optimal Fourier coefficients can be evaluated as

$$
\mathbf{f}_{p, \mathrm{opt}}=\sum_{m=-Q_{h} / 2}^{Q_{h} / 2} \tilde{\mathbf{H}}_{m} \boldsymbol{\gamma}_{(p-m)_{P}}, \quad \text { for } p \in\{0,1, \ldots, P-1\},
$$

with $\boldsymbol{\gamma}_{(p-m)_{P}} \triangleq \mathbf{B}_{(m-p)_{P}} \mathbf{e}_{d} \in \mathbb{C}^{L_{e}+L_{h}+1}$. It is worth noting that $\boldsymbol{y} \triangleq\left[\boldsymbol{\gamma}_{0}^{T}, \boldsymbol{\gamma}_{1}^{T}, \ldots, \boldsymbol{\gamma}_{P-1}^{T}\right]^{T} \in \mathbb{C}^{P\left(L_{e}+L_{h}+1\right)}$ turns out to be the solution of the linear system $\left(\mathscr{H}_{\text {circ }}^{H} \mathscr{H}_{\text {circ }}\right) \boldsymbol{\gamma}=J_{d}$.

In summary, the FRESH design of the optimal LTV-ZF equalizer can be obtained as follows.

Step 1. Given the channel vectors $\left\{\mathbf{h}_{q}(\ell)\right\}_{\ell=0}^{L_{h}}$, for $q \in\left\{-Q_{h} / 2\right.$, $\left.-Q_{h} / 2+1, \ldots, Q_{h} / 2\right\}$, accounting for (5), (6) and (9), construct the matrices $\left\{\tilde{\mathbf{H}}_{q}\right\}_{q=-Q_{h} / 2}^{Q_{h} / 2}$ and, then, build the matrixvalued zero-padded sequence $\left\{\mathscr{H}_{p}\right\}_{p=0}^{P-1}$ in (17).

Step 2. Evaluate the matrices $\left\{\Delta_{p}\right\}_{p=0}^{P-1}$ in (35), by first building the $Q_{h}+1$ matrices $\left\{\mathbf{A}_{q}\right\}_{q=0}^{Q_{h}}$ in (32) and, then, construct the matrices $\left\{\mathbf{B}_{q}\right\}_{q=0}^{P / 2}$ in (36).

\footnotetext{
${ }^{3}$ In the sequel, for the sake of simplicity, we assume that $P$ is an even integer.
}

Step 3. Construct the Fourier coefficients $\left\{\mathbf{f}_{p, \text { opt }}\right\}_{p=0}^{P-1}$ in (37) and, finally, evaluate the equalizer outputs as $y_{\text {opt }}(k)=$ $\psi_{\text {opt }}^{H} \tilde{\mathbf{z}}(k)$, for $k \in \mathcal{K}$.

Some important remarks are now in order. First, although the DFS expansion (16) of the time-varying channel matrix $\tilde{\mathbf{H}}(k)$ is characterized only by $Q_{h}+1$ nonzero matrix-valued coefficients as it is apparent from (17), in general, the DFS expansion (18) of the minimal norm LTV-ZF weight vector $\mathbf{f}_{\text {opt }}(k)$ is instead characterized by a number of nonzero coefficients $\left\{\mathbf{f}_{p, \text { opt }}\right\}_{p=0}^{P-1}$ that are equal to $P$, that is, it depends on the frequency spacing of the complex exponentials employed in the channel model (2). This result is basically due to the fact that the vector $\mathbf{f}_{\text {opt }}(k)$ depends on the channel matrix $\tilde{\mathbf{H}}(k)$ by means of the nonlinear relationship (13). Second, in comparison with its time-domain representation (13), the practical advantage of the FRESH representation is that the time-varying component of the optimal LTV-ZF equalizer consists only of multiplications of the received vector $\mathbf{z}(k)$ by complex exponentials (see Figure 1), whereas the remaining part of the equalizer is predominantly time-invariant. Moreover, it can be observed that, accounting for (35) and (36), the evaluation of the optimal Fourier coefficients (37) involves the calculus of DFT/inverse DFT (IDFT), which can be efficiently computed by using fast Fourier transform (FFT) algorithms. Notwithstanding this, the overall implementation complexity of the FRESH representation of the optimal LTV-ZF equalizer may be quite large for large values of $P$.

\subsection{Low-complexity implementation of the optimal LTV-ZF equalizer}

A simple and direct indication of the implementation complexity of the FRESH representation of the optimal LTV-ZF equalizer is given by the number $P$ of Fourier coefficients employed in the DFS expansion (18) of the time-varying weight vector $\mathbf{f}_{\text {opt }}(k)$, since it determines the number of LTI equalizers to be used in Figure 1. Thus, a significant computational saving can be obtained if the equalizer weight vector is represented with a series expansion by using only a small number of Fourier coefficients. On the basis of this observation, we consider now the problem of optimally approximating $\mathbf{f}_{\text {opt }}(k)$ by means of the following linear combination of only ${ }^{4}$ $Q_{h}+1<Q_{e}+1<P$ complex exponentials:

$$
\mathbf{f}_{\text {subopt }}(k)=\sum_{p=-Q_{e} / 2}^{Q_{e} / 2} \mathbf{f}_{p \text {,subopt }} e^{j(2 \pi / P) p k}=\mathbf{F}_{\text {subopt }} \boldsymbol{\xi}(k),
$$

where $\mathbf{F}_{\text {subopt }} \triangleq\left[\mathbf{f}_{0 \text {,subopt }}, \mathbf{f}_{1, \text { subopt }}, \ldots, \mathbf{f}_{Q_{e} / 2 \text {,subopt }}, \mathbf{f}_{-Q_{e} / 2 \text {,subopt }}\right.$, $\left.\ldots, \mathbf{f}_{-1, \text { subopt }}\right] \in \mathbb{C}^{N\left(L_{e}+1\right) \times\left(Q_{e}+1\right)}$ collects all the coefficients of the series and

$$
\begin{aligned}
\boldsymbol{\xi}(k) \triangleq & {\left[1, e^{j(2 \pi / P) k}, \ldots, e^{j(2 \pi / P)\left(Q_{e} / 2\right) k},\right.} \\
& \left.e^{-j(2 \pi / P)\left(Q_{e} / 2\right) k}, \ldots, e^{-j(2 \pi / P) k}\right]^{T} \in \mathbb{C}^{Q_{e}+1} .
\end{aligned}
$$

\footnotetext{
${ }^{4}$ It is assumed in the sequel that $Q_{e}$ is an even integer number.
} 
A simple and effective criterion to determine $\mathbf{F}_{\text {subopt }}$ consists of minimizing over one period the difference between $\mathbf{f}_{\text {opt }}(k)$ and its approximation $\mathbf{f}_{\text {subopt }}(k)$ in a least squares sense, that is, let $\mathbf{F}$ be an arbitrary matrix belonging to $\mathbb{C}^{N\left(L_{e}+1\right) \times\left(Q_{e}+1\right)}$, the matrix $\mathbf{F}_{\text {subopt }}$ in (38) is chosen as the solution of the following unconstrained optimization problem:

$$
\mathbf{F}_{\text {subopt }}=\arg \min _{\mathbf{F}}\left\{\sum_{k=0}^{P-1}\left\|\mathbf{f}_{\mathrm{opt}}(k)-\mathbf{F} \boldsymbol{\xi}(k)\right\|^{2}\right\} .
$$

It is shown in Appendix B that the solution of the minimization problem (40) is given by

$$
\mathbf{f}_{p, \text { subopt }} \triangleq \begin{cases}\mathbf{f}_{p, \text { opt }}, & \text { for } p \in\left\{0,1, \ldots, Q_{e} / 2\right\}, \\ \mathbf{f}_{P+p, \text { opt }}, & \text { for } p \in\left\{-1,-2, \ldots,-Q_{e} / 2\right\} .\end{cases}
$$

Relation (41) extends to the multidimensional case, a wellknown result encountered in the theory of Fourier series for scalar periodic functions. Specifically, if $\mathbf{f}_{\mathrm{opt}}(k)$ has a Fourier representation, the best approximation $\mathbf{f}_{\text {subopt }}(k)$ using only a reduced number of complex exponentials is obtained by truncating the Fourier series of $\mathbf{f}_{\mathrm{opt}}(k)$ to the desired number of terms. The FRESH implementation of the suboptimal LTV-ZF equalizer $\mathbf{f}_{\text {subopt }}(k)$ is similar to that of its optimal counterpart $\mathbf{f}_{\text {opt }}(k)$, with the important difference that, according to (37), one has to evaluate only $Q_{e}+1$ Fourier coefficients. This implies that, with reference to Figure 1, the FRESH implementation of $\mathbf{f}_{\text {subopt }}(k)$ is composed only by $Q_{e}+1$ LTI equalizers, whose outputs are summed obtaining thus the overall output

$$
\begin{aligned}
y_{\text {subopt }}(k) & =\mathbf{f}_{\text {subopt }}^{H}(k) \mathbf{z}(k)=\left[\sum_{p=-Q_{e} / 2}^{Q_{e} / 2} \mathbf{f}_{p \text { subopt }} e^{j(2 \pi / P) p k}\right]^{H} \mathbf{z}(k) \\
& =\psi_{\text {subopt }}^{H}[\boldsymbol{\xi}(k) \otimes \mathbf{z}(k)], \quad \text { for } k \in \mathcal{K},
\end{aligned}
$$

where $\psi_{\text {subopt }} \triangleq \operatorname{vec}\left(\mathbf{F}_{\text {subopt }}\right) \in \mathbb{C}^{N\left(L_{e}+1\right)\left(Q_{e}+1\right)}$. Given $\psi_{\text {subopt }}$, the implementation complexity, associated with the estimation of each transmitted symbol, involves $N\left(Q_{e}+1\right)\left(L_{e}+1\right)$ multiply-add (MA) complex operations. The ABER performance of the low-complexity LTV-ZF equalizer cannot be evaluated exactly in closed form and will be investigated in Section 6 by Monte Carlo computer simulations.

\section{COMPARISON WITH THE LTV-ZF EQUALIZER PROPOSED IN [9] AND DISCUSSION}

The LTV-ZF equalizer proposed in [9] can be interpreted as a suboptimal version (in the presence of noise) of the FRESH representation of the optimal LTV-ZF equalizer devised in Section 4. The starting point of the approach of [9] is the following series expansion of the equalizer weight vector:

$$
\mathbf{f}_{\mathrm{blm}}(k)=\sum_{p=-Q_{e} / 2}^{Q_{e} / 2} \overline{\mathbf{f}}_{p, \mathrm{blm}} e^{j(2 \pi / P) p k}, \quad \text { for } k \in \mathcal{K},
$$

where only $Q_{h}+1<Q_{e}+1<P$ complex exponentials are employed, which is similar to that considered in (38). In this case, however, the coefficients $\left\{\overline{\mathbf{f}}_{p, \mathrm{blm}}\right\}_{p=-Q_{e} / 2}^{Q_{e} / 2}$ in (43) are chosen so as to satisfy, in the absence of noise, the LTV-ZF condition given by (10). To recast the synthesis of the LTVZF equalizer proposed in [9] in our general framework, we equivalently express the series expansion (43) as follows:

$$
\mathbf{f}_{\mathrm{blm}}(k)=\sum_{p=0}^{P-1} \mathbf{f}_{p, \mathrm{blm}} e^{j(2 \pi / P) p k}, \quad \text { for } k \in \mathbb{Z},
$$

where

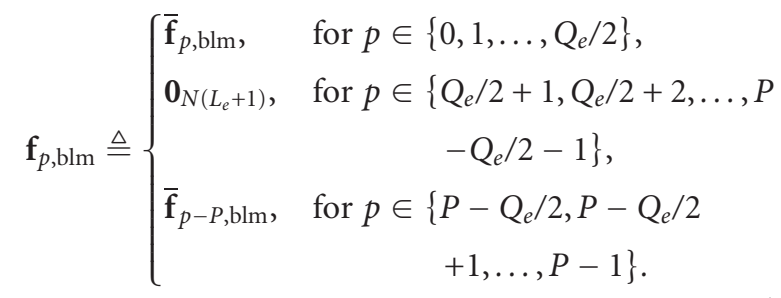

Relation (44) is formally similar to the DFS expansion used in (18) to derive the FRESH representation of $\mathbf{f}_{\text {opt }}(k)$, with the fundamental difference that, in this case, the Fourier coefficients $\left\{\mathbf{f}_{p, \text { blm }}\right\}_{p=Q_{e} / 2+1}^{P-Q_{e} / 2-1}$ are imposed to be identically zero $a$ priori. Based on this observation, let $\psi \triangleq\left[\mathbf{f}_{0}^{T}, \mathbf{f}_{1}^{T}, \ldots, \mathbf{f}_{P-1}^{T}\right]^{T}$ be an arbitrary vector belonging to $\mathbb{C}^{N P\left(L_{e}+1\right)}$; the vector coefficients $\psi_{\text {blm }} \triangleq\left[\mathbf{f}_{0, \text { blm }}^{T}, \mathbf{f}_{1, \text { blm }}^{T}, \ldots, \mathbf{f}_{P-1, \text { blm }}^{T}\right]^{T} \in \mathbb{C}^{N P\left(L_{e}+1\right)}$ used in [9] can be regarded as the solution of the following constrained optimization problem:

$$
\begin{aligned}
& \psi_{\text {blm }}=\arg \min _{\psi}\|\psi\|^{2}, \\
& \text { subject to }\left\{\begin{array}{lr}
\mathscr{H}_{\text {circ }}^{H} \psi=J d, & \mathbf{f}_{p}=\mathbf{0}_{N\left(L_{e}+1\right)}, \\
& \text { for } p \in\left\{\begin{array}{l}
Q_{e} / 2+1, \ldots, P \\
\left.-Q_{e} / 2-1\right\} .
\end{array}\right.
\end{array}\right.
\end{aligned}
$$

Note that, due to the constraints $\mathbf{f}_{p}=\mathbf{0}_{N\left(L_{e}+1\right)}$, for $p \in\left\{Q_{e} / 2+\right.$ $\left.1, \ldots, P-Q_{e} / 2-1\right\}$, the solution $\psi_{\text {blm }}$ differs from $\psi_{\text {opt }}$ given by (27). Accounting for the partition $\mathscr{H}_{\text {circ }}=\left[\left(\mathscr{H}_{\text {circ }}^{(1)}\right)^{T},\left(\mathscr{H}_{\text {circ }}^{(2)}\right)^{T}\right.$, $\left.\left(\mathscr{H}_{\text {circ }}^{(3)}\right)^{T}\right]^{T}$ of the block circulant channel matrix $\mathscr{H}_{\text {circ }}$ given by (22), with

$$
\begin{gathered}
\mathcal{H}_{\text {circ }}^{(1)} \in \mathbb{C}^{N\left(Q_{e} / 2+1\right)\left(L_{e}+1\right) \times P\left(L_{e}+L_{h}+1\right)}, \\
\mathscr{H}_{\text {circ }}^{(2)} \in \mathbb{C}^{N\left(P-Q_{e}-1\right)\left(L_{e}+1\right) \times P\left(L_{e}+L_{h}+1\right)}, \\
\mathscr{H}_{\text {circ }}^{(3)} \in \mathbb{C}^{N\left(Q_{e} / 2\right)\left(L_{e}+1\right) \times P\left(L_{e}+L_{h}+1\right)},
\end{gathered}
$$

the solution of the optimization problem (46) is given by

$$
\bar{\psi}_{\mathrm{blm}}=\left(\mathscr{H}_{\mathrm{blm}}^{H}\right)^{\dagger} \jmath_{d}=\mathscr{H}_{\mathrm{blm}}\left(\mathscr{H}_{\mathrm{blm}}^{H} \mathscr{H}_{\mathrm{blm}}\right)^{-1} \jmath_{d},
$$


where the vector $\bar{\psi}_{\text {blm }} \in \mathbb{C}^{N\left(Q_{e}+1\right)\left(L_{e}+1\right)}$ is obtained from $\psi_{\text {blm }}$ by picking up only its nonzero subvectors and $\mathscr{H}_{\mathrm{blm}}=$ $\left[\left(\mathscr{H}_{\text {circ }}^{(1)}\right)^{T},\left(\mathscr{H}_{\text {circ }}^{(3)}\right)^{T}\right]^{T} \in \mathbb{C}^{N\left(Q_{e}+1\right)\left(L_{e}+1\right) \times P\left(L_{e}+L_{h}+1\right)}$. Finally, accounting for (22), it can be shown that the middle part of $\mathscr{H}_{\text {blm }}$ exhibits $\left(L_{e}+L_{h}+1\right)\left(P-Q_{e}-Q_{h}-1\right)$ zero columns and, thus, the solution (48) can be further simplified as follows:

$$
\bar{\psi}_{\mathrm{blm}}=\overline{\mathscr{H}}_{\mathrm{blm}}\left(\overline{\mathscr{H}}_{\mathrm{blm}}^{H} \overline{\mathscr{H}}_{\mathrm{blm}}\right)^{-1} \bar{\jmath}_{d}
$$

where the matrix $\overline{\mathscr{H}}_{\text {blm }} \in \mathbb{C}^{N\left(Q_{e}+1\right)\left(L_{e}+1\right) \times\left(Q_{e}+Q_{h}+1\right)\left(L_{e}+L_{h}+1\right)}$ is obtained from $\mathscr{H}_{\mathrm{blm}}$ by eliminating its zero columns and $\bar{J}_{d} \triangleq\left[\mathbf{e}_{d}^{T}, \mathbf{0}_{L_{e}+L_{h}+1}^{T}, \ldots, \mathbf{0}_{L_{e}+L_{h}+1}^{T}\right]^{T} \in \mathbb{R}^{\left(Q_{e}+Q_{h}+1\right)\left(L_{e}+L_{h}+1\right)}$.

Some remarks are now in order about the relationships between the LTV-ZF equalizer $\mathbf{f}_{\mathrm{blm}}(k)$ and the optimal LTVZF equalizer $\mathbf{f}_{\text {opt }}(k)$, as well as its low-complexity FRESH version $\mathbf{f}_{\text {subopt }}(k)$.

Remark 1. Because of the additional constraints $\mathbf{f}_{p}=$ $\mathbf{0}_{N\left(L_{e}+1\right)}$, for $p \in\left\{Q_{e} / 2+1, \ldots, P-Q_{e} / 2-1\right\}$ imposed in (46), it follows that $\left\|\psi_{\text {blm }}\right\| \geq\left\|\psi_{\text {opt }}\right\|$ and, consequentially, the LTV-ZF equalizer $\mathbf{f}_{\mathrm{blm}}(k)$ proposed in [9] cannot achieve the minimum ABER in any operative scenario, that is, in both slowly and rapidly time-varying channels. Strictly speaking, although $\mathbf{f}_{\mathrm{blm}}(k)$ exactly satisfies the LTV-ZF condition (10), it is suboptimal in the presence of noise and its ABER performance can be significantly limited by an excessive noise enhancement. On the contrary, the proposed low-complexity LTV-ZF equalizer $\mathbf{f}_{\text {subopt }}(k)$ does not suffer from this limitation since, although it is suboptimal as $\mathbf{f}_{\mathrm{blm}}(k)$, it comes from a least-squares approximation of $\mathbf{f}_{\text {opt }}(k)$. This approach allows one to reduce the implementation complexity of the optimal equalizer $\mathbf{f}_{\text {opt }}(k)$, by assuring a smaller noise amplification.

Remark 2. The LTV-ZF equalizer $\mathbf{f}_{\text {blm }}(k)$ proposed in [9] can be synthesized only if the channel matrix $\overline{\mathscr{H}}_{\text {blm }}$ is full-column rank, that is, $\operatorname{rank}\left(\overline{\mathscr{H}}_{\mathrm{blm}}\right)=\left(Q_{e}+Q_{h}+1\right)\left(L_{e}+L_{h}+1\right)$. Sufficient conditions assuring that $\overline{\mathscr{H}}_{\text {blm }}$ is full-column rank are given in [9], which turn out to be much more restrictive than those provided in Theorem 1 for the synthesis of the optimal LTV-ZF equalizer $\mathbf{f}_{\text {opt }}(k)$. In particular, observe that the condition $\operatorname{rank}\left(\overline{\mathscr{H}}_{\mathrm{blm}}\right)=\left(Q_{e}+Q_{h}+1\right)\left(L_{e}+L_{h}+1\right)$ necessarily requires that $N\left(Q_{e}+1\right)\left(L_{e}+1\right) \geq\left(Q_{e}+Q_{h}+1\right)\left(L_{e}+L_{h}+1\right)$, which leads to using large values of $L_{e}$ and $Q_{e}$ for small values of the number $N$ of antennas, even when the channel is far underspread, that is, $L_{h} Q_{h} \ll P$ (see [9, Figure 4]). In other words, with respect to $\mathrm{f}_{\mathrm{blm}}(k)$, the proposed LTV-ZF equalizers $\mathbf{f}_{\text {opt }}(k)$ and $\mathbf{f}_{\text {subopt }}(k)$ can satisfactorily work by employing smaller values of $L_{e}$ and $Q_{e}$.

Remark 3. Similarly to the low-complexity equalizer $\mathbf{f}_{\text {subopt }} \times$ $(k)$, the LTV-ZF equalizer $\mathbf{f}_{\text {blm }}(k)$ of [9] exhibits a FRESH low-complexity structure, employing only $Q_{e}+1$ LTI equalizers, whose overall output is given by $y_{\mathrm{blm}}(k)=\overline{\boldsymbol{\psi}}_{\mathrm{blm}}^{H}[\boldsymbol{\xi}(k) \otimes$ $\mathbf{z}(k)$ ], for $k \in \mathcal{K}$. Thus, the equalizers $\mathbf{f}_{\mathrm{blm}}(k)$ and $\mathbf{f}_{\text {subopt }}(k)$ exhibit the same implementation complexity. On the other hand, the two equalizers are characterized by different design complexities. The design of $\bar{\psi}_{\text {blm }}$ in (49) entails the direct inversion of the matrix $\overline{\mathscr{H}}_{\text {blm }}^{H} \overline{\mathscr{H}}_{\text {blm }}$, which requires $\mathcal{O}\left[\left(Q_{e}+\right.\right.$ $\left.Q_{h}+1\right)^{3}\left(L_{e}+L_{h}+1\right)^{3}$ ] flops. ${ }^{5}$ As it is apparent from (36) and (37), the complexity associated with the design of the Fourier coefficients $\left\{\mathbf{f}_{p \text {,subopt }}\right\}_{p=-Q_{e} / 2}^{Q_{e} / 2}$ given by (41) is mainly influenced by the evaluation of the vectors $\gamma_{(p-m)_{p}}$, for $p \in$ $\left\{-Q_{e} / 2, \ldots, 0, \ldots, Q_{e} / 2\right\}$ and $m \in\left\{-Q_{h} / 2, \ldots, 0, \ldots, Q_{h} / 2\right\}$; in its turn, for a given value of $p$ and $m$, the design of $\boldsymbol{\gamma}_{(p-m)_{P}}$ is dominated by the inverse FFT (IFFT) of the matrices $\left\{\left(\mathbf{A}_{0}+\Delta_{p}+\Delta_{p}^{H}\right)^{-1}\right\}_{p=0}^{P-1}$; specifically, the inversion of $\left\{\mathbf{A}_{0}+\Delta_{p}+\Delta_{p}^{H}\right\}_{p=0}^{P-1}$ entails $P \cdot \mathcal{O}\left[\left(L_{e}+L_{h}+1\right)^{3}\right]$ flops, whereas the IFFT requires $\left(L_{e}+L_{h}+1\right)^{2} \cdot \mathcal{O}\left(P \log _{2} P\right)$ complex operations. Roughly speaking, since in practice one has that $\log _{2} P \leq L_{e}+L_{h}+1$, it results that the design complexity of the equalizer $\mathbf{f}_{\text {subopt }}(k)$ is less than that of the equalizer proposed in [9] for $P<\left(Q_{e}+Q_{h}+1\right)^{3} /\left[\left(Q_{e}+1\right)\left(Q_{h}+1\right)\right]$. However, it is worth observing from (32) that the circulant matrix $\mathcal{H}_{\text {circ }}^{H} \mathscr{H}_{\text {circ }}$ given by $(31)$ exhibits a large sparse structure; by remembering that the vector $\gamma$ is the solution of the linear system $\left(\mathcal{H}_{\text {circ }}^{H} \mathcal{H}_{\text {circ }}\right) \boldsymbol{\gamma}=J_{d}$, this sparsity structure can be judiciously exploited for computing $\gamma$ by resorting to iterative methods [21]. So doing, a significant reduction of the complexity associated with the design of the subvectors $\boldsymbol{\gamma}_{(p-m)_{P}}$ of $\gamma$ can be obtained, for any value of $P$.

Remark 4. Although $\mathbf{f}_{\text {subopt }}(k)$ represents the best (in the least-squares sense) approximation of $\mathbf{f}_{\text {opt }}(k)$, it satisfies only approximatively the LTV-ZF condition (10) and, thus, unlike the LTV-ZF equalizer proposed in [9], it does not assure perfect symbol recovering in the absence of noise. This is the acceptable price to pay for obtaining a noise-resistant suboptimal LTV-ZF equalizer.

\section{SIMULATION RESULTS}

In this section, the ABER performance of the proposed suboptimal FRESH-LTV-ZF equalizer (referred to as ZF-subopt) is investigated by means of Monte Carlo computer simulations, and compared with the ABER performance of the optimal FRESH-LTV-ZF equalizer (referred to as ZF-opt), as well as with that of the LTV-ZF equalizer proposed in [9] (referred to as ZF-blm).

In all the experiments, the following simulation setting is adopted. The transmitted symbols $s(k)$ and the noise sequences $\left\{v_{n}(k)\right\}_{n=1}^{N}$ are generated according to assumptions (A1) and (A2), and the composite channels $\left\{h_{n}(k, \ell)\right\}_{n=1}^{N}$ are 3 rd-order (i.e., $L_{h}=3$ ) random LTV systems. Specifically, the $n$th channel impulse response $h_{n}(k, \ell)$ is generated as (see, e.g., $[1,14])$

$$
h_{n}(k, \ell)=\frac{1}{\sqrt{M}} \sum_{m=0}^{M-1} \exp \left\{j\left[2 \pi f_{\max } k T_{s} \cos \left(\alpha_{n, m, \ell}\right)\right]+\phi_{n, m, \ell}\right\},
$$

where $M=100$, the random variables $\alpha_{n, m, \ell}$ and $\phi_{n, m, \ell}$ are

\footnotetext{
${ }^{5}$ We refer to [21] for the definition of a floating point operation (flop).
} 
mutually independent over $n, m$, and $\ell$, and uniformly distributed over $[0,2 \pi]$. Observe that, for a given $n$ and $\ell$, relation (50) generates a random process whose power spectrum approximates the Jakes' spectrum arbitrarily well for increasing the value of $M$ (see [1] and references therein). Unless otherwise specified, the doubly selective channel (50) is generated by using the following parameters: carrier frequency $f_{0}=900 \mathrm{MHz}$, symbol period $T_{s}=160 \mu \mathrm{s}$, and mobile speed $v_{\max }=120 \mathrm{~km} / \mathrm{h}$. Thus, the maximum Doppler spread turns out to be $f_{\max }=100 \mathrm{~Hz}$. The block size is equal to $K=50$ and $P=2 K$, which leads to $Q_{h}=2\left\lceil f_{\max } P T_{s}\right\rceil=4$. It should be stressed that the channel model (2) is used only for synthesizing the considered equalizers at the receiver, whereas the received data are generated by resorting to the channel model (50). For each antenna, the BEM coefficients $\left\{h_{q, n}(\ell)\right\}_{q=-Q_{h} / 2}^{Q_{h} / 2}$ are estimated from $h_{n}(k, \ell)$, for all $\ell \in\left\{0,1, \ldots, L_{h}\right\}$, by employing a least-squares algorithm (see [9] for details) and, then, they are used to design the equalizers under comparison. The signal-to-noise ratio (SNR) is defined, according to (1)-(3), as follows (see [14]):

$$
\mathrm{SNR} \triangleq \frac{\left\langle\mathrm{E}\left[\left\|\sum_{\ell=0}^{L_{h}} \mathbf{h}(k, \ell) s(k-\ell)\right\|^{2}\right]\right\rangle_{K}}{\left\langle\mathrm{E}\left[\|\mathbf{v}(k)\|^{2}\right]\right\rangle_{K}},
$$

where $\mathbf{h}(k, \ell) \triangleq\left[h_{1}(k, \ell), h_{2}(k, \ell), \ldots, h_{N}(k, \ell)\right]^{T} \in \mathbb{C}^{N}$. For all the considered equalizers, the equalization delay $d$ is chosen as the integer value nearest to $\left(L_{h}+L_{e}\right) / 2$. All the results are obtained by carrying out $10^{4}$ independent trials, with each run using a different set of data sequences, noise samples, and channel parameters [i.e., $\alpha_{n, m, \ell}$ and $\phi_{n, m, \ell}$ in (50)].

Experiment 1 (ABER versus $Q_{e}$ ). In this experiment, we evaluated the performances of the low-complexity equalizers ZFsubopt and ZF-blm as a function of the number $Q_{e}$ of complex exponential used in the series expansion of their weight vectors $\mathbf{f}_{\text {subopt }}(k)$ in (38) and $\mathbf{f}_{\text {blm }}(k)$ in (43), ranging from 10 to 42 . For comparison, we reported also the performance of the ZF-opt equalizer, whose synthesis does not depend on $Q_{e}$ since it employs all the $P=100$ Fourier coefficients in the DFS expansion of $\mathbf{f}_{\text {opt }}(k)$. The number of receiver antennas is set to $N=N_{\min }=2$, whereas the order of all the considered equalizers is equal to $L_{e}=6$ and, finally, $\mathrm{SNR}=20 \mathrm{~dB}$. Results of Figure 2 evidence that, for $Q_{e} \geq 22$, the proposed ZF-subopt equalizer exhibits performances that are very close to the minimum $\mathrm{ABER}$ of the $\mathrm{ZF}$-opt equalizer and, moreover, significantly outperforms the ZF-blm equalizer [9], especially when small values of $Q_{e}$ are employed. In particular, observe that the proposed ZF-subopt equalizer assures an ABER equal to $10^{-3}$ for a value of $Q_{e}$ as small as 14 , whereas the approach [9] requires about 28 coefficients (i.e., the double) to achieve the same ABER accuracy.

Experiment 2 (ABER versus $L_{e}$ ). In this experiment, we evaluated the performances of the methods under comparison as a function of equalizer order $L_{e}$ ranging $^{6}$ from 4 to 10 . We

\footnotetext{
${ }^{6}$ It is worth noting that, for the simulation setting at hand, both the ZF-
}

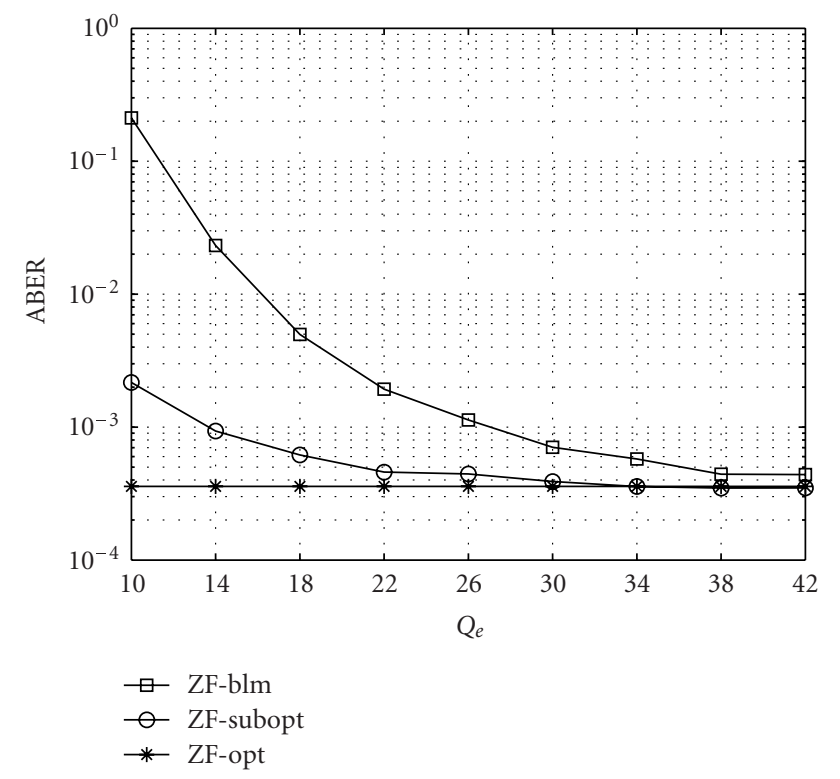

Figure 2: ABER versus $Q_{e}\left(T_{s}=160 \mu \mathrm{s}, f_{\max }=100 \mathrm{~Hz}, N=2\right.$, $L_{e}=6$, and SNR $\left.=20 \mathrm{~dB}\right)$.

considered the same simulation setting of the previous experiment (i.e., $N=2$ and $\mathrm{SNR}=20 \mathrm{~dB}$ ), and we set $Q_{e}=18$ for both the ZF-subopt and ZF-blm equalizers. It can be observed from Figure 3 that the proposed ZF-subopt equalizer exhibits a slight performance degradation with respect to the optimal ZF-opt equalizer, while it significantly outperforms the ZF-blm equalizer for all the considered values of $L_{e}$. Interestingly, it should be observed that, in order to attain the same ABER accuracy, the ZF-blm receiver [9] requires an equalizer order $L_{e}$ that is greater than that of the proposed ZF-subopt method of about two units.

Experiment 3 (ABER versus SNR $(N=2)$ ). In the third experiment, we evaluated the performances of the considered equalizers as a function of SNR ranging from 0 to $25 \mathrm{~dB}$. First, we considered the same simulation setting of the previous experiments (i.e., $N=2, L_{e}=6, Q_{e}=18$ ), whose results are reported in Figure 4 . From this figure, we can observe that, for SNR $\leq 20 \mathrm{~dB}$, the proposed ZF-subopt equalizer again outperforms significantly the ZF-blm equalizer and, moreover, performs very close to the minimal norm ZF-opt equalizer. The performance gain of the ZF-subopt equalizer with respect to the ZF-blm equalizer becomes less pronounced when the SNR approaches $25 \mathrm{~dB}$. As previously announced in Remark 4, this behavior stems from the fact that, unlike the ZF-blm equalizer, the ZF-subopt equalizer does not assure perfect symbol recovery in the absence of noise; however, our simulation results (not reported here)

opt and ZF-subopt equalizers can work with $L_{e} \geq 2$ (see condition (C1)); in contrast, the ZF-blm equalizer proposed in [9] does not exist [i.e., the matrix $\overline{\mathscr{H}}_{\text {blm }}$ in (49) cannot be full-column rank] for $L_{e} \in\{2,3\}$. 


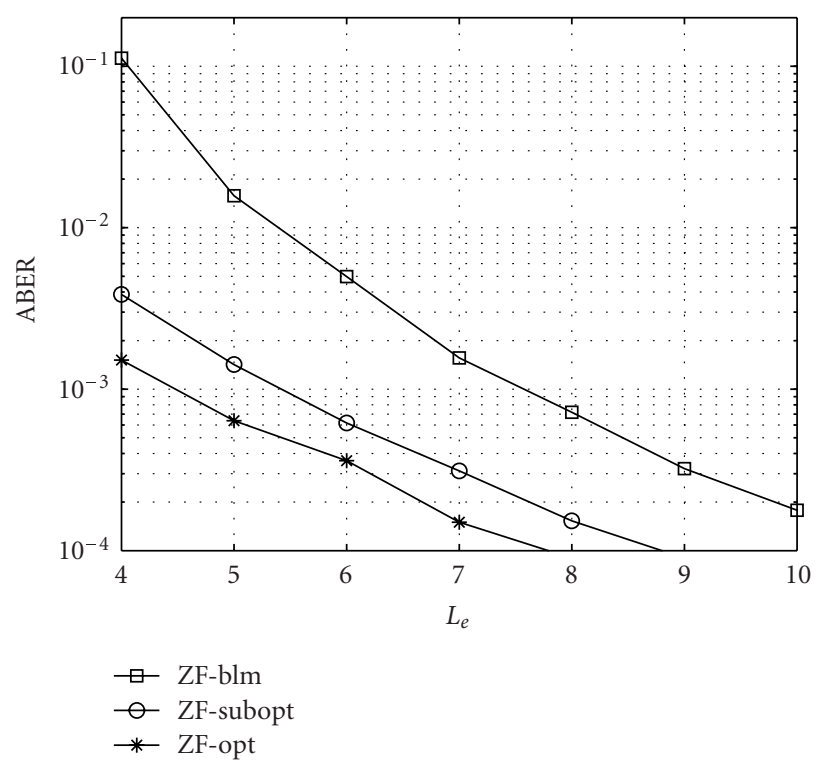

FIgURE 3: ABER versus $L_{e}\left(T_{s}=160 \mu \mathrm{s}, f_{\max }=100 \mathrm{~Hz}, N=2, Q_{e}=\right.$ 18 , and $\mathrm{SNR}=20 \mathrm{~dB})$.

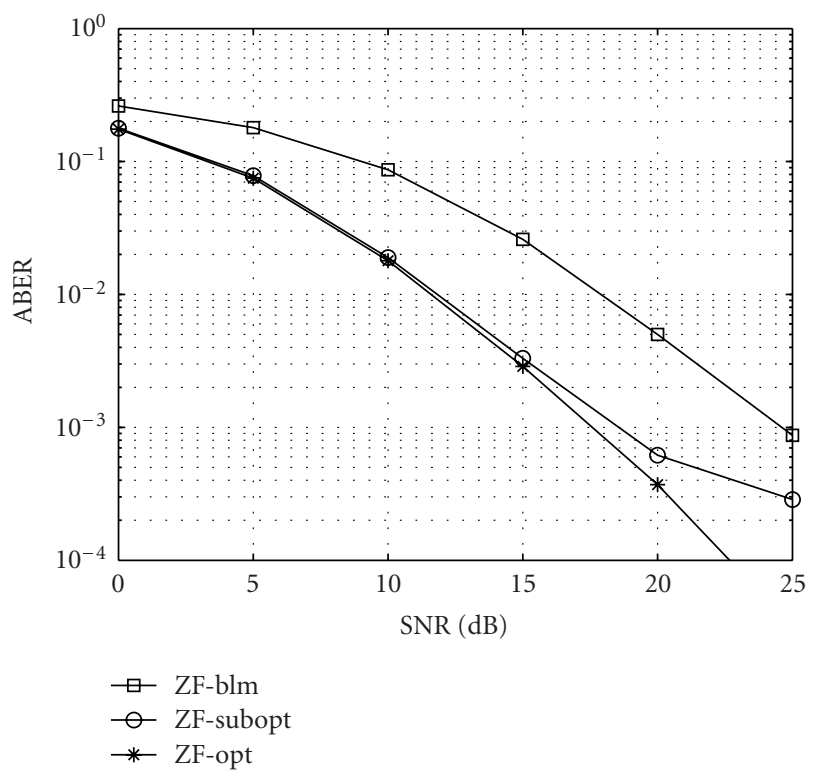

Figure 4: ABER versus SNR $\left(T_{s}=160 \mu \mathrm{s}, f_{\max }=100 \mathrm{~Hz}, N=2\right.$, $L_{e}=6$, and $\left.Q_{e}=18\right)$.

show that the ZF-blm equalizer outperforms the ZF-subopt one only for large values of the SNR, which are beyond the range of practical interest.

Experiment 4 (ABER versus SNR $(N=3)$ ). To further corroborate the performances of the equalizers under comparison, we considered in Figure 5 a different simulation setting. Specifically, we employed at the receiver $N=3$ antennas, and we set $Q_{e}=18$ (as for the results of Figure 5) and $L_{e}=2$. Results of Figure 5 show that, by increasing

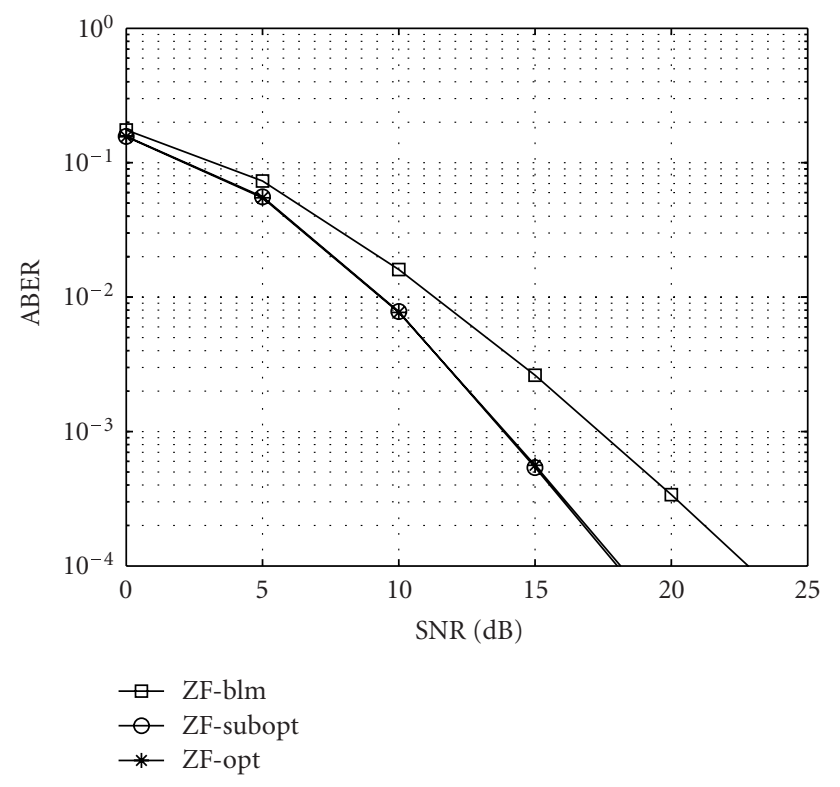

FIGURE 5: ABER versus SNR $\left(T_{s}=160 \mu \mathrm{s}, f_{\max }=100 \mathrm{~Hz}, N=3\right.$, $L_{e}=2$, and $\left.Q_{e}=18\right)$.

the number of receiver antennas, all the considered equalizers improve their performances and, remarkably, the proposed ZF-subopt equalizer performs very close to the ZFopt equalizer, assuring ABER values smaller than $10^{-4}$ for SNR $>15 \mathrm{~dB}$.

Experiment 5 (ABER versus SNR $(N=2$, different environment)). In the last experiment, we considered a more rapidly time-varying environment, wherein the doubly selective channel (50) is generated by using the following parameters: carrier frequency $f_{0}=3.6 \mathrm{GHz}$, symbol period $T_{s}=50 \mu \mathrm{s}$, and mobile speed $v_{\max }=240 \mathrm{~km} / \mathrm{h}$. Thus, the maximum Doppler spread turns out to be $f_{\max }=800 \mathrm{~Hz}$. The block size is set equal to $K=50$ and $P=2 K$, which leads to $Q_{h}=2\left\lceil f_{\max } P T_{s}\right\rceil=8$. As for the rest, we considered the same simulation setting of Experiment 2 (i.e., $N=2$ and SNR $=20 \mathrm{~dB}$ ) and we set $Q_{e}=22$ for both the ZFsubopt and ZF-blm equalizers. Results of Figure 6 confirm all the aforementioned considerations, by showing in particular that, in comparison with the previous environment, the ZF-blm equalizer pays a greater performance penalty with respect to both the ZF-opt and ZF-subopt equalizers.

\section{CONCLUSIONS}

We have considered the problem of synthesizing LTV-ZF equalizers for both time- and frequency-selective channels. Relying on a BEM of the rapidly time-varying channel

\footnotetext{
${ }^{7}$ Note that, for the simulation setting at hand, unlike the ZF-subopt equalizer, the ZF-blm equalizer cannot work with $Q_{e}=18$.
} 


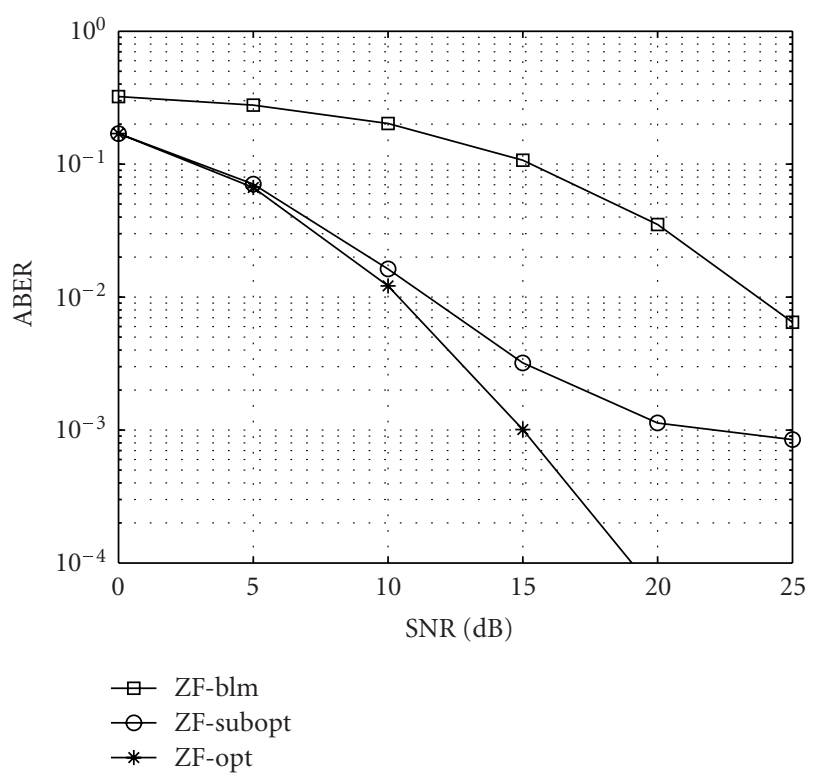

FIgURE 6: ABER versus SNR $\left(T_{s}=50 \mu \mathrm{s}, f_{\max }=800 \mathrm{~Hz}, N=2\right.$, $L_{e}=6$, and $Q_{e}=22$ ).

impulse response, we have derived the canonical frequencydomain representation of the minimal norm LTV-ZF equalizer, which assures the best ABER performances in the presence of AWGN, and we have discussed the mathematical conditions assuring its existence. The frequency-domain representation of the optimal LTV-ZF equalizer has the advantage that, unlike its time-domain counterpart, exhibits many similarities to time-invariant filtering, wherein the time-varying component of the equalizer consists of computing FRESH versions of the received data vector. On the basis of this FRESH representation, we have proposed a low-complexity LTV-ZF equalizer, whose synthesis is based on a truncated DFS expansion of the equalizer weight vector, and, moreover, we have provided an alternative interpretation of the LTV-ZF equalizer recently proposed in [9]. A comparative study between the three LTV-ZF approaches has shown that, for all the SNR values of practical interest, the proposed lowcomplexity LTV-ZF equalizer is able to significantly outperform the equalizer [9], especially when a limited number $Q_{e}$ of Fourier coefficients are used in the truncated DSF expansion and/or small values of the equalizer order $L_{e}$ are employed.

\section{APPENDICES}

\section{A. PROOF OF THEOREM 1}

Let us preliminarily characterize the null space $\mathcal{N}[\tilde{\mathbf{H}}(k)]$ of the matrix $\tilde{\mathbf{H}}(k)$ defined in (10). For a given $k \in \mathbb{Z}$, an arbitrary nonnull vector $\xi \in \mathbb{C}^{L_{e}+L_{h}+1}$ belongs to the subspace $\mathcal{N}[\tilde{\mathbf{H}}(k)]$ if and only if

$$
\tilde{\mathbf{H}}(k) \boldsymbol{\xi}=\sum_{q=-Q_{h} / 2}^{Q_{h} / 2}\left[\tilde{\mathbf{H}}_{q} \boldsymbol{\xi}\right] e^{j(2 \pi / P) q k}=\mathbf{0}_{N\left(L_{e}+1\right)} .
$$

Since the complex exponentials in (A.1) are linearly independent functions, the previous equation holds if and only if $\tilde{\mathbf{H}}_{q} \boldsymbol{\xi}=\mathbf{0}_{N\left(L_{e}+1\right)}$, for all $q \in\left\{-Q_{h} / 2,-Q_{h} / 2+1, \ldots, Q_{h} / 2\right\}$. In other words, a vector $\boldsymbol{\xi} \neq \mathbf{0}_{L_{e}+L_{h}+1}$ belongs to the subspace $\mathcal{N}[\tilde{\mathbf{H}}(k)]$ if and only if

$$
\xi \in \bigcap_{q=-Q_{h} / 2}^{Q_{h} / 2} \mathcal{N}\left(\tilde{\mathbf{H}}_{q}\right)
$$

Thus, if condition (C1) holds, the matrix $\tilde{\mathbf{H}}(k)$ turns out to be full-column rank, for all $k \in \mathbb{Z}$, if and only if the null spaces of the matrices $\left\{\tilde{\mathbf{H}}_{q}\right\}_{q=-Q_{h} / 2}^{Q_{h} / 2}$ intersect only trivially, that is,

$$
\bigcap_{q=-Q_{h} / 2}^{Q_{h} / 2} \mathcal{N}\left(\widetilde{\mathbf{H}}_{q}\right)=\left\{\mathbf{0}_{L_{e}+L_{h}+1}\right\}
$$

Since, by virtue of (5), $\tilde{\mathbf{H}}_{q}=\mathbf{J}_{q} \mathbf{H}_{q}$ and the block diagonal matrices $\left\{\mathbf{J}_{q}\right\}_{q=-Q_{h} / 2}^{Q_{h} / 2}$ are nonsingular, it follows that $\mathcal{N}\left(\tilde{\mathbf{H}}_{q}\right)=$ $\mathcal{N}\left(\mathbf{H}_{q}\right)$, for all $q \in\left\{-Q_{h} / 2,-Q_{h} / 2+1, \ldots, Q_{h} / 2\right\}$. At this point, we rely on a well-known identifiability result (see, e.g., [22]) encountered in blind equalization of SIMO FIR systems. Specifically, for a given $q \in\left\{-Q_{h} / 2,-Q_{h} / 2+1\right.$, $\left.\ldots, Q_{h} / 2\right\}$, let $H_{q, n}(z) \triangleq \sum_{\ell=0}^{L_{h}} h_{q, n}(\ell) z^{-\ell}$ denote the $N$ channel transfer functions associated with the sequences $\left\{h_{q, n}(\ell)\right\}_{\ell=0}^{L_{h}}$, for $n \in\{1,2, \ldots, N\}$, the null space $\mathcal{N}\left(\mathbf{H}_{q}\right)$ of the block Toeplitz matrix $\mathbf{H}_{q}$ is empty if the polynomials $H_{q, 1}(z), H_{q, 2}(z), \ldots, H_{q, N}(z)$ have no common zeros. On the other hand, if the channel transfer functions $H_{q, 1}(z), H_{q, 2}(z), \ldots, H_{q, N}(z)$ have $0<M_{q} \leq L_{h}$ common zeros $z_{q, 1}, z_{q, 2}, \ldots, z_{q, M_{q}}$, then $\mathcal{N}\left(\mathbf{H}_{q}\right)$ is spanned by the $M_{q}$ generalized Vandermonde vectors [22] corresponding to $z_{q, 1}, z_{q, 2}, \ldots, z_{q, M_{q}}$. On the basis of this result, we can infer that, if $z_{q_{1}, n_{1}} \neq z_{q_{2}, n_{2}}$, for all $q_{1} \neq q_{2} \in\left\{-Q_{h} / 2,-Q_{h} / 2+\right.$ $\left.1, \ldots, Q_{h} / 2\right\}$ and for all $n_{1} \neq n_{2} \in\{1,2, \ldots, N\}$, then condition (A.3) is surely satisfied and, thus, $\operatorname{rank}[\tilde{\mathbf{H}}(k)]=L_{e}+L_{h}+$ 1 , for all $k \in \mathbb{Z}$.

\section{B. CALCULUS OF THE MATRIX $\mathrm{F}_{\text {subopt }}$}

The object function $\mathcal{L}(\mathbf{F}) \triangleq \sum_{k=0}^{P-1}\left\|\mathbf{f}_{\text {opt }}(k)-\mathbf{F} \boldsymbol{\xi}(k)\right\|^{2}$ of the problem (40) can be written as

$$
\begin{gathered}
\mathcal{L}(\mathbf{F})=\sum_{k=0}^{P-1}\left[\left\|\mathbf{f}_{\mathrm{opt}}(k)\right\|^{2}-\mathbf{f}_{\mathrm{opt}}^{H}(k) \mathbf{F} \boldsymbol{\xi}(k)-\boldsymbol{\xi}^{H}(k) \mathbf{F}^{H} \mathbf{f}_{\mathrm{opt}}(k)\right. \\
\left.+\boldsymbol{\xi}^{H}(k) \mathbf{F}^{H} \mathbf{F} \boldsymbol{\xi}(k)\right] .
\end{gathered}
$$

By neglecting the terms that do not depend on $\mathbf{F}$ and invoking the properties of the trace operator [19], one obtains that minimizing the object function (B.1) is equivalent to 
minimizing the following one:

$$
\mathcal{L}^{\prime}(\mathbf{F})=\operatorname{trace}\left(\mathbf{F} \boldsymbol{\Omega} \mathbf{F}^{H}\right)-\operatorname{trace}(\mathbf{F} \boldsymbol{\Sigma})-\operatorname{trace}\left(\boldsymbol{\Sigma}^{H} \mathbf{F}^{H}\right),
$$

where

$$
\begin{gathered}
\boldsymbol{\Omega} \triangleq \sum_{k=0}^{P-1} \boldsymbol{\xi}(k) \boldsymbol{\xi}^{H}(k) \in \mathbb{C}^{\left(Q_{e}+1\right) \times\left(Q_{e}+1\right)}, \\
\boldsymbol{\Sigma} \triangleq \sum_{k=0}^{P-1} \boldsymbol{\xi}(k) \mathbf{f}_{\mathrm{opt}}^{H}(k) \in \mathbb{C}^{\left(Q_{e}+1\right) \times N\left(L_{e}+1\right)} .
\end{gathered}
$$

The matrix $\mathbf{F}_{\text {subopt }}$ minimizing the object function (B.2) must satisfy [23] the matrix equation $\mathbf{F}_{\text {subopt }} \boldsymbol{\Omega}=\boldsymbol{\Sigma}^{H}$, which can be decomposed in $Q_{e}+1$ vector equalities as follows:

$$
\mathbf{F}_{\text {subopt }} \sum_{k=0}^{P-1} \boldsymbol{\xi}(k) e^{-j \omega_{p} k}=\sum_{k=0}^{P-1} \mathbf{f}_{\mathrm{opt}}(k) e^{-j \omega_{p} k},
$$

for $p \in\left\{-Q_{e} / 2,-Q_{e} / 2+1, \ldots, Q_{e} / 2\right\}$, where $\omega_{p}=(2 \pi / P) p$ if $p \in\left\{0,1, \ldots, Q_{e} / 2\right\}$, else $\omega_{p}=(2 \pi / P)(P+p)$ if $p \in\{-1,-2$, $\left.\ldots,-Q_{e} / 2\right\}$. Since all the entries of vector $\sum_{k=0}^{P-1} \xi(k) e^{-j \omega_{p} k}$ are zero, except for its $\left[(p)_{Q_{e}+1}+1\right]$ th component that is equal to $P$, one has

$$
\mathbf{f}_{p, \text { subopt }}=\frac{1}{P} \sum_{k=0}^{P-1} \mathbf{f}_{\mathrm{opt}}(k) e^{-j \omega_{p} k}
$$

for $p \in\left\{-Q_{e} / 2,-Q_{e} / 2+1, \ldots, Q_{e} / 2\right\}$, which, by definition, is equal to $\mathbf{f}_{p \text {,opt }}$ if $p \in\left\{0,1, \ldots, Q_{e} / 2\right\}$, else is equal to $\mathbf{f}_{p+P \text {,opt }}$ if $p \in\left\{-1,-2, \ldots,-Q_{e} / 2\right\}$.

\section{ACKNOWLEDGMENTS}

The author wishes to acknowledge with gratitude Professor G. Gelli for his careful reading of the manuscript, and the anonymous reviewers for their constructive comments and suggestions. This work is partially supported by Italian National project Wireless $8 \mathrm{O} 2.16$ Multiantenna mEsh Networks (WOMEN) under Grant number 2005093248.

\section{REFERENCES}

[1] G. B. Giannakis and C. Tepedelenlioğlu, "Basis expansion models and diversity techniques for blind identification and equalization of time-varying channels," Proceedings of the IEEE, vol. 86, no. 10, pp. 1969-1986, 1998.

[2] M. K. Tsatsanis and G. B. Giannakis, "Modeling and equalizing of rapidly fading channels," International Journal of Adaptive Control and Signal Processing, pp. 159-176, 1998.

[3] A. M. Sayeed and B. Aazhang, "Joint multipath-doppler diversity in mobile wireless communications," IEEE Transactions on Communications, vol. 47, no. 1, pp. 123-132, 1999.

[4] X. Ma and G. B. Giannakis, "Maximum-diversity transmissions over doubly selective wireless channels," IEEE Transac- tions on Information Theory, vol. 49, no. 7, pp. 1832-1840, 2003.

[5] G. Leus, S. Zhou, and G. B. Giannakis, "Orthogonal multiple access over time- and frequency-selective channels," IEEE Transactions on Information Theory, vol. 49, no. 8, pp. 19421950, 2003.

[6] G. Leus, I. Barhumi, and M. Moonen, "Low-complexity serial equalization of doubly-selective channels," in Proceedings of the 6th Baiona Workshop on Signal Processing in Communications, pp. 69-74, Baiona, Spain, September 2003.

[7] C. Tepedelenlioğlu and G. B. Giannakis, "Transmitter redundancy for blind estimation and equalization of time- and frequency-selective channels," IEEE Transactions on Signal Processing, vol. 48, no. 7, pp. 2029-2043, 2000.

[8] W. C. Jakes, Microwave Mobile Channels, John Wiley \& Sons, New York, NY, USA, 1974.

[9] I. Barhumi, G. Leus, and M. Moonen, "Time-varying FIR equalization for doubly selective channels," IEEE Transactions on Wireless Communications, vol. 4, no. 1, pp. 202-214, 2005.

[10] J. K. Tugnait and W. Luo, "Linear prediction error method for blind identification of periodically time-varying channels," IEEE Transactions on Signal Processing, vol. 50, no. 12, pp. 3070-3082, 2002.

[11] L. Franks, "Polyperiodic linear filtering," in Cyclostationarity in Communications and Signal Processing, W. A. Gardner, Ed., pp. 240-266, IEEE Press, Piscataway, NJ, USA, 1994.

[12] A. Ben-Israel and T. N. E. Greville, Generalized Inverses, Springer, New York, NY, USA, 2002.

[13] P. A. Bello, "Characterization of randomly time-variant channels," IEEE Transactions on Communications, vol. 11, no. 4, pp. 360-393, 1963.

[14] J. K. Tugnait and W. Luo, "Blind identification of time-varying channels using multistep linear predictors," IEEE Transactions on Signal Processing, vol. 52, no. 6, pp. 1739-1749, 2004.

[15] W. Luo and J. K. Tugnait, "Semi-blind time-varying channel estimation using superimposed training," in Proceedings of International Conference on Acoustics, Speech, and Signal Processing (ICASSP '04), pp. 797-800, Montreal, Canada, May 2004.

[16] G. Leus, "On the estimation of rapidly time-varying channels," in Proceedings of the European Signal Processing Conference, pp. 120-123, Vienna, Austria, September 2004.

[17] J. G. Proakis, Digital Communications, McGraw-Hill, New York, NY, USA, 2001.

[18] L. Tong and S. Perreau, "Multichannel blind identification: from subspace to maximum likelihood methods," Proceeedings of the IEEE, vol. 86, no. 10, pp. 1951-1968, 1998.

[19] R. A. Horn and C. R. Johnson, Matrix Analysis, Cambridge University Press, Cambridge, UK, 1990.

[20] R. Vescovo, "Inversion of block-circulant matrices and circular array approach," IEEE Transactions on Antennas and Propagation, vol. 45, no. 10, pp. 1565-1567, 1997.

[21] A. Björck, Numerical Methods for Least Squares Problems, SIAM, Philadelphia, Pa, USA, 1996.

[22] E. Serpedin and G. B. Giannakis, "A simple proof of a known blind channel identifiability result," IEEE Transactions on Signal Processing, vol. 47, no. 2, pp. 591-593, 1999.

[23] M. Honig, U. Madhow, and S. Verdù, "Blind adaptive multiuser detection," IEEE Transactions on Information Theory, vol. 41, no. 4, pp. 944-960, 1995. 
Francesco Verde was born in Santa Maria Capua Vetere, Italy, on June 12, 1974. He received the Dr. Eng. degree (summa cum laude) in electronic engineering in 1998 from the Second University of Napoli, and the Ph.D. degree in information engineering in 2002, from the University of Napoli Federico II. Since 2002, he has been an Assistant Professor with the Department of Electronic and Telecommunication Engi-

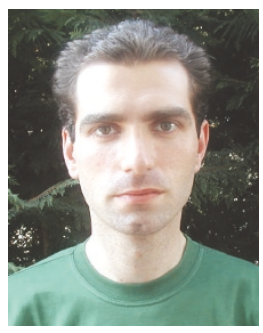
neering, University of Napoli Federico II. His research activities lie in the areas of statistical signal processing, digital communications, and communication systems. In particular, his current interests are focused on cyclostationarity-based techniques for blind identification, equalization, and interference suppression for narrowband modulation systems, code-division multiple-access systems, and multicarrier modulation systems. 